\title{
Symmetries of Cosmological Cauchy Horizons *
}

\author{
Vincent Moncrief ${ }^{1}$ and James Isenberg ${ }^{2}$ \\ 1 Department of Physics, Yale University, P.O. Box 6666, New Haven, CT 06511, USA \\ 2 Department of Mathematics, University of Oregon, Eugene, OR 97403, USA
}

\begin{abstract}
We consider analytic vacuum and electrovacuum spacetimes which contain a compact null hypersurface ruled by closed null generators. We prove that each such spacetime has a non-trivial Killing symmetry. We distinguish two classes of null surfaces, degenerate and non-degenerate ones, characterized by the zero or non-zero value of a constant analogous to the "surface gravity" of stationary black holes. We show that the non-degenerate null surfaces are always Cauchy horizons across which the Killing fields change from spacelike (in the globally hyperbolic regions) to timelike (in the acausal, analytic extensions).

For the special case of a null surface diffeomorphic to $\mathbf{T}^{3}$ we characterize the degenerate vacuum solutions completely. These consist of an infinite dimensional family of "plane wave" spacetimes which are entirely foliated by compact null surfaces. Previous work by one of us has shown that, when one dimensional Killing symmetries are allowed, then infinite dimensional families of non-degenerate, vacuum solutions exist. We recall these results for the case of Cauchy horizons diffeomorphic to $\mathbf{T}^{3}$ and prove the generality of the previously constructed non-degenerate solutions.

We briefly discuss the possibility of removing the assumptions of closed generators and analyticity and proving an appropriate generalization of our main results. Such a generalization would provide strong support for the cosmic censorship conjecture by showing that causality violating, cosmological solutions of Einstein's equations are essentially an artefact of symmetry.
\end{abstract}

\section{Introduction}

As is well known, there exist vacuum solutions of Einstein's equations such as the Taub-NUT solutions which contain smooth, compact Cauchy horizons. These horizons separate globally hyperbolic regions (e.g., Taub space) from causality

* Research supported in part by NSF grant No. PHY79-16482 at Yale and No. PHY79-13146 at Berkeley 
violating regions which contain closed timelike lines (e.g., NUT or Newman-UntiTamburino space). The two parameter family of Taub solutions are all spacially homogeneous and thus very special, but one can construct infinite parameter families of inhomogeneous vacuum spacetimes with qualitatively similar properties $[1,2]$. In [2] for example, we showed how to construct an infinite dimensional family of vacuum spacetimes which each have a compact Cauchy horizon diffeomorphic to $\mathbf{T}^{3}$ with closed null generators and which in general have only a single Killing vector field. One can extend this construction to obtain an infinite parameter generalization of the Taub-NUT solutions for which the Cauchy horizons are all diffeomorphic to $\mathbf{S}^{3}$ and are always Killing horizons with closed generators [3]. Each of these solutions has a single Killing vector field which is space-like in the globally hyperbolic region, null on the horizon (and thus tangent to its null generators) and timelike in the causality violating extension (where its orbits become closed timelike curves). These families of extendible spacetimes with one Killing field are rather large, having (roughly speaking) half the dimension of the space of all solutions of the same symmetry type.

The existence of such large families of Taub-NUT-like solutions suggests the danger of a counter-example to the strong cosmic censorship conjecture [4]. Can one remove the symmetry restriction and construct still larger families of extendible, causality violating spacetimes? Do there exist open sets of such spacetimes within the space of all vacuum solutions? If so, then cosmic censorship is false.

The purpose of this paper is to show that the assumption of a Killing symmetry was crucial to constructing solutions of the type described above. More precisely, we shall show that analytic, vacuum (or electrovacuum) spacetimes with compact null surfaces ruled (in a local product bundle fashion) by closed null orbits necessarily have a Killing symmetry. The Killing field implied by the theorem is always tangent to the generators of the compact null surface in question. In the non-degenerate case (a term we shall define properly in Sect. III) the null surface is always a Cauchy horizon and the Killing field is always spacelike in the globally hyperbolic region and timelike in the extension. In the degenerate case the null surface need not be a Cauchy horizon at all. In Sect. IV we shall exhibit an infinite dimensional family of degenerate solutions on $\mathbf{T}^{3} \times \mathbf{R}$ which each have a compact null surface through every event.

The assumption of analyticity is probably not crucial to the main argument but is used here to simplify the analysis. More important is the topological constraint that the generators of the null surfaces form closed orbits (with a local, but not necessarily global, product bundle structure). However, we believe that even this condition may be removable and that only compactness of the null surfaces (and satisfaction of the field equations) is essential. We shall return to this question briefly in the concluding section.

The plan of this paper is as follows. In Sect. II we describe more precisely the family of spacetimes we propose to study and introduce convenient coordinate charts on neighborhoods of their compact null surfaces. In Sect. III we prove our main theorem on the existence of Killing symmetries. In Sect. IV we discuss the degenerate solutions for the special case of null surfaces diffeomorphic to $\mathbf{T}^{3}$ and characterize these solutions completely. We also show that the non-degenerate 
solutions on $\mathbf{T}^{3} \times \mathbf{R}$ are precisely those constructed (in the sense of convergent power series) explicitly in [2]. Finally, in Sect. V we discuss some possible generalizations of our result and their potential relevance to the cosmic censorship conjecture.

\section{Mathematical Preliminaries}

\section{A. Geometrical Assumptions}

Let ${ }^{(4)} V=M \times \mathbf{R}$, where $M$ is a compact three manifold (without boundary) and let $g$ be a Lorentz metric on ${ }^{(4)} V$. We want to consider such spacetimes which are time orientable and which have compact null embedded hypersurfaces diffeomorphic to $M$ with closed (null geodesic) generators. More precisely, we want to consider null hypersurfaces which have a local product bundle structure in the following sense. If $N$ is such a hypersurface and $\gamma$ is a closed null generator of $N$, then there exists an open set $U_{\gamma} \mathrm{C}^{(4)} V$ containing $\gamma$ such that

(i) $U_{\gamma} \cap N$ is diffeomorphic to $B_{\gamma} \times \mathbf{S}^{1}$ for some two-manifold $B_{\gamma}$ and some diffeomorphism $\phi_{\gamma}: U_{\gamma} \cap N \rightarrow B_{\gamma} \times \mathbf{S}^{1}$, and

(ii) there is a smooth, surjective map $\pi_{\gamma}: B_{\gamma} \times \mathbf{S}^{1} \rightarrow B_{\gamma}$ such that, for any $p \in B_{\gamma}$, $B_{\gamma} \times \mathbf{S}^{1} \approx B_{\gamma} \times \pi_{\gamma}^{-1}(p)$ and the fiber $\pi_{\gamma}^{-1}(p)$ is diffeomorphic (via $\phi_{\gamma}^{-1}$ ) to a closed null generator lying in $U_{\gamma} \cap N$.

In other words, $\phi_{\gamma}^{-1}$ embeds $\pi_{\gamma}^{-1}(p)$ as a closed submanifold of $U_{\gamma} \cap N$ coinciding with a null generator of $N$ for each $p \in B_{\gamma}$.

We shall refer to submanifolds of the type $U_{\gamma} \cap N$ as elementary regions of $N$. By definition, each elementary region is a product bundle with fibers diffeomorphic to $\mathbf{S}^{1}$ and coinciding with null generators of $N$. By compactness, $N$ can be covered by a finite number of such elementary regions.

These assumptions imply that if we introduce coordinates $\left\{x^{a}, x^{3}\right\}$ on $B_{\gamma} \times \mathbf{S}^{1}$ such that $\left\{x^{a}\right\} \equiv\left\{x^{1}, x^{2}\right\}$ are constant along the fibers and such that $x^{3}$ is a periodic coordinate on the circle (with, say, period $2 \pi$ ), then we can use these (via $\phi_{\gamma}$ ) as coordinates for $U_{\gamma} \cap N$ with the property that the $\left\{x^{a}\right\}$ are constant along the null generators of $U_{\gamma} \cap N$. Note that we do not require that the $x^{3}$ coordinate be geared to any particular parametrization of the null generators lying in $U_{\gamma} \cap N$. Ultimately we shall find that there is a "natural" parameterization of these generators defined by the action of a Killing field on $N$. We shall then be able to "glue" the elementary regions together in such a way as to regard $N$ as a principal $\mathrm{U}(1)$ bundle over a suitable quotient manifold. $A$ priori, however, no such preferred U(1) action on $N$ is assumed to exist.

Several examples may help to clarify the range of possibilities covered by the above assumptions. The simplest case is that in which $N$ and its null generators form a global product bundle, i.e., $N$ is diffeomorphic to $B \times \mathbf{S}^{1}$ for some compact two manifold $B$ and there exists a smooth projection $\pi: N \rightarrow B$ whose fibers coincide (as closed submanifolds $\approx \mathbf{S}^{1}$ ) with the null generators of $N$. Examples of this type were constructed in [1,2] for the special case $N \approx \mathbf{T}^{3} \approx \mathbf{T}^{2} \times \mathbf{S}^{1}$. More generally, one can consider non-trivial circle bundles which only locally have the product structure discussed above. For example, the horizons of the Taub-NUT solutions are diffeomorphic to $\mathbf{S}^{3}$ and their null generators coincide with the fibers 
of a Hopf fibration $\pi: \mathbf{S}^{3} \rightarrow \mathbf{S}^{2}$. As we mentioned earlier, one can construct an infinite parameter family of vacuum spacetimes with this topologically non-trivial horizon structure [3].

If $\left\{x^{a}, x^{3}\right\}$ are coordinates defined on an elementary region $U_{y} \cap N$ of the type described above, then there are many ways to extend these to coordinates $\left\{t, x^{a}, x^{3}\right\}$ on a neighborhood $\tilde{U}_{\gamma} \approx \mathbf{R} \times\left(U_{\gamma} \cap N\right)$ of $U_{\gamma} \cap N$ such that $U_{\gamma} \cap N$ corresponds to the level surface $t=0$ and such that the (locally defined) vector field $\frac{\partial}{\partial x^{3}}$ has closed integral curves on $\tilde{U}_{\gamma}$. A particularly convenient choice for such a construction is defined in the following subsection.

Since $N$ is compact, one can cover a full neighborhood of $N$ in ${ }^{(4)} V$ with a finite number of such developments of elementary regions in $N$. In any such local coordinate chart the Lorentz metric $g$ has the properties that

$$
\begin{gathered}
\left.g_{33}\right|_{t=0}=\left.g_{3 a}\right|_{t=0}=0, \\
\left.g_{t 3}\right|_{t=0} \neq 0, \quad a=1,2,
\end{gathered}
$$

and that $\left.g_{a b}\right|_{t=0}$ is positive definite. The first two conditions ensure that $N$ is null and that $\left.\frac{\partial}{\partial x^{3}}\right|_{N}$ is tangent to its generators, whereas the last two conditions are needed for the non-degeneracy and Lorentz signature of $g$. Our basic analyticity assumption is that

(iii) $g$ is analytic in a family of charts of the type described above which cover a neighborhood of $N$ in ${ }^{(4)} V$. If Maxwell fields are considered, then the field tensor $F=F_{\mu \nu} d x^{\mu} \wedge d x^{\nu}$ is also analytic.

\section{B. Coordinate Conditions}

Given an elementary region in $N$ we wish to construct new coordinates on a neighborhood of this region which simplify the form of the metric. We shall follow a procedure somewhat analogous to the construction of gaussian normal coordinates but adapted to the null character of $N$. Define a null vector field $\left.\tilde{k}\right|_{N}$ throughout the elementary region by the algebraic conditions

$$
\begin{gathered}
\left.k^{\mu} k^{v} g_{\mu \nu}\right|_{t=0}=0, \\
\left.k^{\mu} g_{\mu 3}\right|_{t=0}=1,\left.\quad k^{\mu} g_{\mu a}\right|_{t=0}=0 .
\end{gathered}
$$

It is straightforward to solve these equations explicitly and show that $\left.\tilde{k}\right|_{N}$ is analytic, nowhere zero and everywhere transversal to the elementary region (having $\left.k^{t}\right|_{t=0}$ nowhere zero). Now for each point $p$ in the elementary region, construct the unique, affinely parametrized, null geodesic through $p$ with the initial conditions $(p, \tilde{k}(p))$. Define coordinates on a neighborhood of the elementary region by the requirements that the "spacial" coordinates $\left\{x^{3^{\prime}}, x^{a^{\prime}}\right\}$ are constant along the null geodesics so constructed and that the "time" coordinate $t^{\prime}$ vanishes on $N$ and coincides with the affine parameter time along each of the transversal null geodesics. 
In such a coordinate system the metric takes on the simplified form

$$
g=2 d t^{\prime} d x^{3^{\prime}}+\phi\left(d x^{3^{\prime}}\right)^{2}+2 \beta_{a} d x^{a^{\prime}} d x^{3^{\prime}}+\mu_{a b} d x^{a^{\prime}} d x^{b^{\prime}}
$$

where $\mu_{a b}$ is positive definite near $t^{\prime}=0$, and where

$$
\left.\phi\right|_{t^{\prime}=0}=\left.\beta_{a}\right|_{t^{\prime}=0}=0 .
$$

Because of their similarity to gaussian normal coordinates, we shall refer to such systems as gaussian null coordinates.

An alternative construction which leads to the same metric form (and also demonstrates analyticity of the coordinate transformation) is to regard the coordinate conditions

$$
\begin{aligned}
g_{t^{\prime} t^{\prime}}=0 & =\frac{\partial x^{\alpha}}{\partial t^{\prime}} \frac{\partial x^{\beta}}{\partial t^{\prime}} g_{\alpha \beta}, \\
g_{t^{\prime} 3^{\prime}}=1 & =\frac{\partial x^{\alpha}}{\partial t^{\prime}} \frac{\partial x^{\beta}}{\partial x^{3^{\prime}}} g_{\alpha \beta}, \\
g_{t^{\prime} a^{\prime}}=0 & =\frac{\partial x^{\alpha}}{\partial t^{\prime}} \frac{\partial x^{\beta}}{\partial x^{a^{\prime}}} g_{\alpha \beta},
\end{aligned}
$$

as an evolutionary system for the new coordinate functions $\left\{t^{\prime}, x^{3^{\prime}}, x^{a^{\prime}}\right\}\left(t, x^{3}, x^{a}\right)$. One solves Eqs. (2.5) algebraically for the time derivatives $\frac{\partial x^{\alpha^{\prime}}}{\partial t}$ (using $\frac{\partial x^{\alpha}}{\partial x^{\mu^{\prime}}} \frac{\partial x^{\mu^{\prime}}}{\partial x^{\beta}}$ $=\delta_{\beta}^{\alpha}$, etc., to define $\frac{\partial x^{\alpha}}{\partial x^{\mu^{\prime}}}$ in terms of $\left.\frac{\partial x^{\mu^{\prime}}}{\partial x^{\alpha}}\right)$ and proves the local existence of analytic solutions of the resulting system by means of the Cauchy-Kowalewski theorem, taking

$$
\begin{gathered}
t^{\prime}\left(0, x^{3}, x^{a}\right)=0, \\
x^{3^{\prime}}\left(0, x^{3}, x^{a}\right)=x^{3}, \\
x^{a^{\prime}}\left(0, x^{3}, x^{a}\right)=x^{a},
\end{gathered}
$$

as initial conditions.

Somewhat more generally one can take the alternative initial conditions

$$
\begin{gathered}
t^{\prime}\left(0, x^{3}, x^{a}\right)=0, \\
x^{3^{\prime}}\left(0, x^{3}, x^{a}\right)=h\left(x^{3}, x^{a}\right), \\
x^{a^{\prime}}\left(0, x^{3}, x^{a}\right)=f^{a}\left(x^{b}\right),
\end{gathered}
$$

where $\operatorname{det}\left|\frac{\partial f^{a}}{\partial x^{b}}\right| \neq 0, \frac{\partial h}{\partial x^{3}} \neq 0$, and where $x^{3^{\prime}}\left(0, x^{3}, x^{a}\right)=h\left(x^{3}, x^{a}\right)$ is a new periodic coordinate function (with period $2 \pi$ ) on each null generator $x^{a}=$ constant (i.e., $\frac{\partial h}{\partial x^{3}}$ is non-vanishing and periodic in $x^{3}$ for each $x^{a}$ and $\left.h\left(x^{3}+2 \pi, x^{a}\right)=h\left(x^{3}, x^{a}\right)+2 \pi\right)$. 
These more general initial conditions allow us to change the labeling of the null generators and to change the parameterization of each null generator independently in an essentially arbitrary (analytic) fashion.

One should keep in mind that gaussian null coordinates are geometrically less "natural" than gaussian normal coordinates since they are non-trivially geared to the choice of coordinates in the initial surface. Thus, for example, a change of initial conditions from the form (2.6) to the form (2.7) will, in general, change the family of transversal null geodesics along which the $\left\{x^{3^{\prime}}, x^{a^{\prime}}\right\}$ are constant and change the "time" function $t^{\prime}$ defined by the construction.

\section{Field Equations}

We present here, for subsequent reference, the Einstein-Maxwell equations expressed in a gaussian null coordinate chart $\left\{t, x^{3}, x^{a}\right\}$. The metric has the form

$$
g=2 d t d x^{3}+\phi\left(d x^{3}\right)^{2}+2 \beta_{a} d x^{a} d x^{3}+\mu_{a b} d x^{a} d x^{b},
$$

where $\phi$ and $\beta_{a}$ vanish at the null surface $t=0$, and where $\mu_{a b}$ is [for each fixed $\left.\left(t, x^{3}\right)\right]$ a Riemannian two-metric near $t=0$. We shall follow the sign conventions of Misner et al. [5] and, in addition, choose units such that $G=c=1$.

The components of the Ricci tensor are given by

$$
\begin{aligned}
R_{t t}= & -\frac{1}{2} \mu^{a b} \mu_{a b, t t}+\frac{1}{4} \mu^{a c} \mu^{b d} \mu_{a b, t} \mu_{c d, t}, \\
R_{t 3}= & \frac{1}{\sqrt{\mu}}\left[\sqrt{\mu}\left(\frac{1}{2} \phi_{, t}-\frac{1}{2} \beta^{a} \beta_{a, t}\right)\right]_{, t}+\frac{1}{\sqrt{\mu}}\left[\sqrt{\mu}\left(\frac{\mu^{a b}}{2} \beta_{a, t}\right)\right]_{, b} \\
& -\frac{1}{2} \mu^{a b} \mu_{a b, t 3}+\frac{1}{4} \mu^{a c} \mu^{b d} \mu_{a b, t} \mu_{c d, 3}, \\
R_{t b}= & \frac{1}{\sqrt{\mu}}\left[\sqrt{\mu}\left(\frac{1}{2} \beta_{b, t}-\frac{1}{2} \beta^{a} \mu_{a b, t}\right)\right]_{, t}+{ }^{(2)} \nabla_{c}\left(\frac{1}{2} \mu^{a c} \mu_{a b, t}\right)-{ }^{(2)} \nabla_{b}\left(\frac{1}{2} \mu^{c d} \mu_{c d, t}\right), \\
R_{33}= & \frac{1}{\sqrt{\mu}}\left[\sqrt{\mu}\left(\frac{1}{2} \phi_{, 3}+\frac{1}{2} \phi \phi_{, t}+\frac{1}{2} \beta^{a} \phi_{, a}-\beta^{a} \beta_{a, 3}-\frac{1}{2} \beta^{a} \beta_{a} \phi_{, t}\right)\right]_{, t} \\
& +\frac{1}{\sqrt{\mu}}\left[\sqrt{\mu} \mu^{a c}\left(-\frac{\phi_{, a}}{2}+\beta_{a, 3}+\frac{\beta_{a}}{2} \phi_{, t}\right)\right]_{, c} \\
& -\frac{1}{\sqrt{\mu}}\left[\sqrt{\mu} \phi_{, t}\right]_{, 3}-\frac{1}{2} \mu^{a b} \mu_{a b, 33}+\frac{1}{4} \mu^{a c} \mu^{b d} \mu_{a b, 3} \mu_{c d, 3} \\
& -\left\{\frac{1}{2}\left(\phi_{, t}-\beta^{a} \beta_{a, t}\right)^{2}+\frac{1}{4} \mu^{a c} \mu^{b d}\left(\beta_{a, b}-\beta_{b, a}\right)\left(\beta_{d, c}-\beta_{c, d}\right)\right. \\
& \left.+\frac{1}{2} \mu^{d c} \beta_{c, t}\left(2 \phi_{, d}+\phi \beta_{d, t}-2 \beta^{a}\left(\beta_{a, d}-\beta_{d, a}\right)-2 \beta_{d, 3}-\beta^{a} \beta_{a} \beta_{d, t}\right)\right\}, \\
R_{3 b}= & \frac{1}{\sqrt{\mu}}\left[\sqrt{\mu}\left(\frac{1}{2} \phi_{, b}+\frac{1}{2} \phi \beta_{b, t}-\frac{1}{2} \beta^{a}\left(\beta_{a, b}-\beta_{b, a}\right)-\frac{1}{2} \beta^{a} \beta_{a} \beta_{b, t}-\frac{1}{2} \beta^{a} \mu_{a b, 3}\right)\right]_{, t} \\
& +{ }^{(2)} \nabla_{c}\left[\frac{\mu^{a c}}{2}\left(\beta_{a, b}-\beta_{b, a}\right)+\frac{1}{2} \beta^{c} \beta_{b, t}+\frac{1}{2} \mu^{a c} \mu_{a b, 3}\right]
\end{aligned}
$$




$$
\begin{aligned}
& -\frac{1}{\sqrt{\mu}}\left[\sqrt{\mu} \frac{\beta_{b, t}}{2}\right]_{, 3}-\frac{1}{2} \mu^{c d}{ }^{(2)} \nabla_{b}\left(\mu_{c d, 3}\right) \\
& -\left\{\frac{1}{2}\left(\beta_{b, t}-\beta^{a} \mu_{a b, t}\right)\left(\phi, t-\beta^{c} \beta_{c, t}\right)\right. \\
& +\frac{1}{2} \mu^{d c} \mu_{c b, t}\left(\phi_{, d}+\phi \beta_{d, t}-\beta^{a}\left(\beta_{a, d}-\beta_{d, a}\right)-\beta_{d, 3}-\beta^{a} \beta_{a} \beta_{d, t}\right) \\
& \left.+\frac{1}{2} \mu^{a c} \beta_{a, t}\left(-\mu_{b c, 3}+{ }^{(2)} \nabla_{c} \beta_{b}\right)\right\} \\
R_{a b}= & \frac{1}{\sqrt{\mu}}\left[\sqrt{\mu}\left(-\frac{1}{2} \mu_{a b, 3}+\frac{1}{2}\left({ }^{(2)} \nabla_{b} \beta_{a}+{ }^{(2)} \nabla_{a} \beta_{b}\right)+\frac{\phi}{2} \mu_{a b, t}-\frac{1}{2} \beta^{c} \beta_{c} \mu_{a b, t}\right)\right]_{, t} \\
& +{ }^{(2)} \nabla_{c}\left[\frac{\beta^{c}}{2} \mu_{a b, t}\right]-\frac{1}{\sqrt{\mu}}\left[\sqrt{\mu} \frac{\mu_{a b, t}}{2}\right]_{, 3} \\
& +{ }^{(2)} R_{a b}-\left\{\frac{1}{2}\left(\beta_{a, t}-\beta^{c} \mu_{a c, t}\right)\left(\beta_{b, t}-\beta^{d} \mu_{b d, t}\right)\right. \\
& +\frac{1}{4} \mu^{d f} \mu_{f b, t}\left(-2 \mu_{a d, 3}+2^{(2)} \nabla_{d} \beta_{a}+\left(\phi-\beta^{g} \beta_{g}\right) \mu_{a d, t}\right) \\
& \left.+\frac{1}{4} \mu^{d f} \mu_{f a, t}\left(-2 \mu_{b d, 3}+2^{(2)} \nabla_{d} \beta_{b}+\left(\phi-\beta^{g} \beta_{g}\right) \mu_{b d, t}\right)\right\},
\end{aligned}
$$

where $\mu^{a b}$ is the inverse of $\mu_{a b}, \beta^{a}=\mu^{a b} \beta_{b},{ }^{(2)} R_{a b}$ is the Ricci tensor of $\mu_{a b}$ and ${ }^{(2)} \nabla_{a}$ represents covariant differentiation with respect to this metric. In applying the operator ${ }^{(2)} \nabla_{a}$, one treats $\phi, \phi_{, t}, \phi, 3$ as scalars, $\beta_{a}, \beta_{a, t}, \beta_{a, 3}$ as covariant vectors and $\mu_{a b}, \mu_{a b, t}, \mu_{a b, 3}$ as second rank tensors respectively $\left[\right.$ e.g. ${ }^{(2)} \nabla_{b}\left(\beta_{a, t}\right)=\partial_{b}\left(\beta_{a, t}\right)$ ${ }^{(2)} \Gamma_{a b}^{c} \beta_{c, t}$, where ${ }^{(2)} \Gamma_{b c}^{a}$ are the Christoffel symbols of $\left.\mu_{a b}\right]$. Finally, $\mu=\operatorname{det}\left(\mu_{a b}\right)$.

When Maxwell fields are included, the field tensor, $F=F_{\mu \nu} d x^{\mu} \wedge d x^{\nu}$, satisfies

$$
F_{[\mu \nu, \gamma]}=0,
$$

and

$$
{ }^{(4)} \nabla_{\mu} F^{\mu v}=0 \text {, }
$$

where

$$
\begin{aligned}
{ }^{(4)} \nabla_{\beta} F^{t \beta}= & \frac{1}{\sqrt{\mu}}\left(\sqrt{\mu} \beta^{a} F_{t a}\right)_{, 3}+\frac{1}{\sqrt{\mu}}\left(\sqrt{\mu} F_{3 t}\right)_{, 3}+\frac{1}{\sqrt{\mu}} \\
& \cdot\left[\sqrt{\mu}\left(\left(\beta^{a} \beta_{a}-\phi\right) \mu^{b c} F_{t c}+\beta^{b} F_{t 3}+\mu^{a b} F_{3 a}+\beta^{a} \beta^{b} F_{a t}+\mu^{b c} \beta^{a} F_{c a}\right)\right]_{, b}, \\
{ }^{(4)} \nabla_{\beta} F^{3 \beta}= & \frac{1}{\sqrt{\mu}}\left(\sqrt{\mu} F_{t 3}\right)_{, t}+\frac{1}{\sqrt{\mu}}\left(\sqrt{\mu} \beta^{a} F_{a t}\right)_{, t}+\frac{1}{\sqrt{\mu}}\left(\sqrt{\mu} \mu^{a b} F_{t a}\right)_{, b}, \\
{ }^{(4)} \nabla_{\beta} F^{a \beta}= & \frac{1}{\sqrt{\mu}}\left[\sqrt{\mu}\left(\mu^{a c} F_{c 3}+\mu^{a c} \beta^{d} F_{d c}+\mu^{a c}\left(\beta^{d} \beta_{d}-\phi\right) F_{c t}+\beta^{a} F_{3 t}+\beta^{a} \beta^{c} F_{t c}\right)\right]_{, t} \\
& +\frac{1}{\sqrt{\mu}}\left[\sqrt{\mu} \mu^{a c} F_{c t}\right]_{, 3}+\frac{1}{\sqrt{\mu}}\left[\sqrt{\mu}\left(\mu^{a c} \mu^{b d} F_{c d}+\mu^{a c} \beta^{b} F_{t c}+\beta^{a} \mu^{b c} F_{c t}\right)\right]_{, b} .
\end{aligned}
$$

Finally, since the Maxwell stress energy tensor has zero trace, Einstein's equations become

$$
R_{\mu \nu}=8 \pi T_{\mu \nu},
$$


where

$$
\begin{gathered}
8 \pi T_{t t}=2 F_{t a} F_{t b} \mu^{a b}, \\
8 \pi T_{t 3}=-2\left(F_{t 3}\right)^{2}+2 F_{t a} F_{t 3} \beta^{a}+2 F_{t a} F_{3 b} \mu^{a b}-\frac{1}{2} F_{\mu v} F^{\mu v}, \\
8 \pi T_{t b}=2 F_{t 3} F_{b t}+2 F_{t a} F_{b c} \mu^{a c}+2 F_{t b} F_{t a} \beta^{a}, \\
8 \pi T_{33}=2 F_{3 a} F_{3 b} \mu^{a b}-\frac{1}{2} \phi F_{\mu v} F^{\mu v}-4 F_{3 a} F_{3 t} \beta^{a}+2\left(F_{3 t}\right)^{2}\left(\beta^{a} \beta_{a}-\phi\right), \\
8 \pi T_{3 b}=2 F_{3 t} F_{b t}\left(\beta^{a} \beta_{a}-\phi\right)+2 F_{3 t} F_{b 3} \\
-2 F_{3 t} F_{b a} \beta^{a}-2 F_{3 a} F_{b t} \beta^{a}+2 F_{3 a} F_{b c} \mu^{a c}-\frac{1}{2} \beta_{b} F_{\mu v} F^{\mu v}, \\
8 \pi T_{a b}=2 F_{a 3} F_{b t}+2 F_{b 3} F_{a t}+2 F_{a t} F_{b t}\left(\beta^{c} \beta_{c}-\phi\right)-2 F_{a t} F_{b c} \beta^{c} \\
-2 F_{b t} F_{a c} \beta^{c}+2 F_{a c} F_{b d} \mu^{c d}-\frac{1}{2} \mu_{a b} F_{\mu v} F^{\mu \nu}
\end{gathered}
$$

in which

$$
\begin{aligned}
F_{\mu v} F^{\mu v}= & -2\left(F_{t 3}\right)^{2}+4 F_{t 3} F_{t a} \beta^{a}+4 F_{3 b} F_{t a} \mu^{a b}-4 F_{a b} F_{t c} \mu^{b c} \beta^{a} \\
& +F_{a b} F_{c d} \mu^{a c} \mu^{b d}-2 F_{t a} F_{t b} \beta^{a} \beta^{b}+2 F_{t a} F_{t b} \mu^{a b}\left(\beta^{c} \beta_{c}-\phi\right) .
\end{aligned}
$$

\section{Existence of a Killing Symmetry}

In this section we prove our main result: that analytic vacuum (and electrovacuum) spacetimes with compact null hypersurfaces of the type defined in Sect. II always have a Killing symmetry. We shall be led to distinguish two subclasses of such compact null surfaces, non-degenerate and degenerate surfaces. The non-degenerate hypersurfaces are always Cauchy horizons which separate globally hyperbolic open submanifolds of spacetime from their causality violating, analytic extensions. The degenerate null hypersurfaces, on the other hand, need not be Cauchy horizons at all. We shall characterize the degenerate solutions completely for the special case ${ }^{(4)} V \approx \mathbf{T}^{3} \times \mathbf{R}$ when we return to this question in Sect. IV.

To simplify the statement of the proof we shall discuss the vacuum case by itself. We shall then describe the slight modifications that are needed to encompass the electrovacuum case.

\section{A. Local Arguments}

A key step in the argument will entail a change of coordinates within an (arbitrary) elementary region of $N$ and the construction of a new (gaussian null) coordinate system on a neighborhood of that elementary region. We shall distinguish the new (gaussian null) coordinates and metric functions from the old by a supercript "prime" ('). Thus we shall write $\left(t^{\prime}, x^{3^{\prime}}, x^{a^{\prime}}\right), \phi^{\prime}, \beta_{a}^{\prime}, \mu_{a b}^{\prime}$, etc. for the transformed quantities. Furthermore, we shall (wherever convenient) designate the restriction of a function to the null hypersurface $t=t^{\prime}=0$ by an overhead "nought" $\left({ }^{\circ}\right)$ writing, for example, $\stackrel{\circ}{a b}_{a b}, \stackrel{\leftrightarrow}{\phi}_{, t}$, etc., for $\left.\mu_{a b}\right|_{t=0}$ and $\left.\phi_{, t}\right|_{t=0}$.

We first note that the Einstein equation $R_{33}=0$ restricts to

$$
\stackrel{\circ}{R}_{33}=0=-\left.\left[(\ln \sqrt{\mu})_{, 33}+\frac{1}{2} \phi_{, t}(\ln \sqrt{\mu})_{, 3}+\frac{1}{4} \mu^{a c} \mu^{b d} \mu_{a b, 3} \mu_{c d, 3}\right]\right|_{t=0},
$$


where $\mu \equiv \operatorname{det}\left(\mu_{a b}\right)$. This is essentially Raychaudhuri's equation for the null generators of $N$. Since $\mu_{a b}$ must be smooth on each null generator, we may apply the maximum principle [6] to Eq. (3.1) (regarded as an elliptic equation on $\mathbf{S}^{1}$ for $\ln \sqrt{\mu}$ ) to prove that $\stackrel{\circ}{\mu}_{, 3}=0$ and thus, again from Eq. (3.1) that $\stackrel{\circ}{\mu}_{a b, 3}=0$. Thus in any gaussian null coordinate system on any elementary region of $N, \mu_{a b}$ is constant along the null generators.

Now restrict the equation $R_{3 b}=0$ to $t=0$ and use the fact that $\stackrel{\circ}{\mu}_{a b, 3}=0$ to derive the equation

$$
\left.\left(\phi, t b-\beta_{b, t 3}\right)\right|_{t=0}=0 \text {. }
$$

Integrate this equation with respect to $x^{3}$ and appeal to the smoothness of $\dot{\beta}_{b, t}$ to show that

$$
\frac{\partial}{\partial x^{b}}\left(\int_{0}^{2 \pi} d x^{3} \dot{\phi}_{, t}\right)=0
$$

and thus that $\int_{0}^{2 \pi} d x^{3} \dot{\phi}_{, t}$ is constant on an elementary region. Let us designate this constant by $2 \pi k$ so that

$$
\int_{0}^{2 \pi} d x^{3} \stackrel{\circ}{\phi}, t^{2}=2 \pi k=\text { constant }
$$

throughout the given elementary region. We shall find later that $k$ does not vary from one elementary region to another.

We now show that one can introduce new coordinates on an elementary region and corresponding new gaussian null coordinates on a neighborhood of this region such that the transformed quantity ${\stackrel{\circ}{\phi}, t^{\prime}}^{\prime}$ is constant throughout the elementary region and has the value $k$ defined above. To accomplish this consider a coordinate transformation of the form

$$
\begin{gathered}
x^{3^{\prime}}=h\left(x^{3}, x^{a}\right), \\
x^{a^{\prime}}=x^{a},
\end{gathered}
$$

and construct, as described in Sect. IIB, the associated new gaussian null coordinates $\left(t^{\prime}, x^{3^{\prime}}, x^{a^{\prime}}\right)$ on a neighborhood of the original elementary region. In general, this neighborhood will be different from that covered by the original gaussian null coordinates $\left(t, x^{3}, x^{a}\right)$ though, of course, the two neighborhoods will have the same intersection with $N\left(\right.$ at $\left.t=t^{\prime}=0\right)$. By writing out the transformation equations, one can evaluate the quantity $\left.\phi_{, t^{\prime}}^{\prime}\right|_{t^{\prime}=0}$ in terms of the original metric functions and the (as yet unspecified) transformation function $h$. The result is :

$$
2 \frac{\partial}{\partial x^{3}}\left(\frac{\partial h}{\partial x^{3}}\right)+\left(\frac{\partial h}{\partial x^{3}}\right) \dot{\phi}_{, t}-\left(\frac{\partial h}{\partial x^{3}}\right)^{2}{\stackrel{\circ}{\phi}, t^{\prime}}^{\prime}=0
$$

which is a Riccati equation for the function $\frac{\partial h}{\partial x^{3}}$.

Equation (3.6) can be derived in a slightly different way which illuminates its geometrical significance. Recall that the acceleration $\tilde{a}$ of a parameterized curve, 
defined in any coordinate chart by

$$
a^{\mu}=\frac{d^{2} x^{\mu}}{d \lambda^{2}}+\Gamma_{\alpha \beta}^{\mu} \frac{d x^{\alpha}}{d \lambda} \frac{d x^{\beta}}{d \lambda}
$$

obeys the covariance relation

$$
a^{\mu^{\prime}}=\frac{\partial x^{\mu^{\prime}}}{\partial x^{\alpha}} a^{\alpha}
$$

In any gaussian null coordinate system $\left(t, x^{3}, x^{a}\right)$, a curve satisfying $t(\lambda)=0, x^{a}(\lambda)$ $=$ constant has an acceleration given by

$$
\begin{gathered}
a^{t}=a^{a}=0, \\
a^{3}=\frac{d^{2} x^{3}}{d \lambda^{2}}-\frac{\stackrel{\phi}{\phi}, t_{2}}{2}\left(\frac{d x^{3}}{d \lambda}\right)^{2} .
\end{gathered}
$$

The covariance relation (3.8) therefore leads, for a transformation of the type considered, to

$$
\frac{d^{2} x^{3^{\prime}}}{d \lambda^{2}}-\frac{\stackrel{\circ}{\phi}_{, t^{\prime}}^{\prime}}{2}\left(\frac{d x^{3^{\prime}}}{d \lambda}\right)^{2}=\frac{\partial x^{3^{\prime}}}{\partial x^{3}}\left[\frac{d^{2} x^{3}}{d \lambda^{2}}-\frac{\stackrel{\circ}{\phi}, t_{2}}{2}\left(\frac{d x^{3}}{d \lambda}\right)^{2}\right]
$$

Substituting the transformation $x^{3^{\prime}}=h\left(x^{3}, x^{a}\right)$ into this equation leads directly to the Riccati equation (3.6).

We wish to set $\dot{\phi}^{\prime}, t^{\prime}=k=$ constant and show that Eq. (3.6) has an analytic solution $u=\frac{\partial h}{\partial x^{3}}$ which defines a new periodic coordinate function $x^{3^{\prime}}$ throughout the elementary region. We therefore set ${\stackrel{\circ}{\phi^{\prime}},}_{t^{\prime}}^{\prime}=k, u=\frac{\partial h}{\partial x^{3}}$ and rewrite Eq. (3.6) as

$$
\frac{\partial u}{\partial x^{3}}+\left(\frac{\stackrel{\phi}{, t}}{2}\right) u-\left(\frac{k}{2}\right) u^{2}=0
$$

and recall that the value of $k$ is given by Eq. (3.4).

We can solve Eq. (3.11) explicitly but we must distinguish the two cases, $k=0$ (the degenerate case) and $k \neq 0$ (the non-degenerate case). First consider the nondegenerate case and assume for definiteness that $k>0$.

Defining

$$
p\left(x^{3}, x^{a}\right)=\exp \left(-\int_{0}^{x^{3}} d \tilde{x}^{3} \frac{\AA_{, t}}{2}\left(\tilde{x}^{3}, x^{a}\right)\right),
$$

and recalling Eq. (3.4), we see that

$$
p\left(x^{3}+2 \pi, x^{a}\right)=\exp (-\pi k) p\left(x^{3}, x^{a}\right), \forall x^{3} \in \mathbf{R}, \quad p\left(0, x^{a}\right)=1,
$$

where we regard $\dot{\phi}_{, t}\left(x^{3}, x^{a}\right)$ as a periodic function of $x^{3}$ (with period $2 \pi$ ) defined for all $x^{3} \in \mathbf{R}$.

We claim that the appropriate solution of Eq. (3.11) is given by 


$$
\begin{aligned}
u\left(x^{3}, x^{a}\right) & =\frac{\partial h\left(x^{3}, x^{a}\right)}{\partial x^{3}} \\
& =+\frac{2}{k} \frac{p\left(x^{3}, x^{a}\right)}{\left[\frac{\int_{0}^{2 \pi} d y\left(p\left(y, x^{a}\right)\right)}{1-e^{-\pi k}}\right]-\int_{0}^{x^{3}} d y\left(p\left(y, x^{a}\right)\right)} \\
& =-\frac{2}{k} \frac{\partial}{\partial x^{3}} \ln \left[\frac{\int_{0}^{2 \pi} d y\left(p\left(y, x^{a}\right)\right)}{1-e^{-\pi k}}-\int_{0}^{x^{3}} d y\left(p\left(y, x^{a}\right)\right)\right]
\end{aligned}
$$

To justify this we verify directly that

(i) $u\left(x^{3}, x^{a}\right)=\frac{\partial h\left(x^{3}, x^{a}\right)}{\partial x^{3}}$ is an analytic, nowhere vanishing solution of Eq. (3.11) for all $x^{3} \in \mathbf{R}$,

(ii) $\frac{\partial h\left(x^{3}, x^{a}\right)}{\partial x^{3}}$ is periodic in $x^{3}$ with period $2 \pi$ and has the integral property $h\left(x^{3}+2 \pi, x^{a}\right)-h\left(x^{3}, x^{a}\right)=2 \pi$.

The proof of (i) is facilitated by noting that the quantity

$$
D\left(x^{3}, x^{a}\right) \equiv\left[\frac{\int_{0}^{2 \pi} d y\left(p\left(y, x^{a}\right)\right)}{1-e^{-\pi k}}\right]-\int_{0}^{x^{3}} d y\left(p\left(y, x^{a}\right)\right)
$$

satisfies, by virtue of Eq. (3.13),

$$
D\left(x^{3}+2 \pi, x^{a}\right)=e^{-\pi k} D\left(x^{3}, x^{a}\right),
$$

$\forall x^{3} \in \mathbf{R}$ and thus never vanishes for all $x^{3} \in \mathbf{R}$ (since clearly $D\left(x^{3}, x^{a}\right)>0$, $\left.\forall x^{3} \in(-\infty, 2 \pi]\right)$.

The periodicity of $\frac{\partial h\left(x^{3}, x^{a}\right)}{\partial x^{3}}=u\left(x^{3}, x^{a}\right)$ follows from (3.13) and (3.16), which give

$$
u\left(x^{3}+2 \pi, x^{a}\right)=\frac{2}{k} \frac{p\left(x^{3}+2 \pi, x^{a}\right)}{D\left(x^{3}+2 \pi, x^{a}\right)}=\frac{2}{k} \frac{p\left(x^{3}, x^{a}\right)}{D\left(x^{3}, x^{a}\right)}=u\left(x^{3}, x^{a}\right) .
$$

To complete the proof of (ii), in view of the periodicity of $u$, it suffices to note that

$$
\begin{aligned}
h\left(2 \pi, x^{a}\right)-h\left(0, x^{a}\right) & =-\left.\frac{2}{k} \ln \left[\frac{\int_{0}^{2 \pi} d y\left(p\left(y, x^{a}\right)\right)}{1-e^{-\pi k}}-\int_{0}^{x^{3}} d y\left(p\left(y, x^{a}\right)\right)\right]\right|_{0} ^{2 \pi} \\
& =-\frac{2}{k} \ln \left[e^{-\pi k}\right]=2 \pi .
\end{aligned}
$$

These results show that any transformation of the form

$$
x^{3^{\prime}}=g\left(x^{a}\right)+\ln \left[D\left(x^{3}, x^{a}\right)^{-2 / k}\right], \quad x^{a^{\prime}}=x^{a},
$$


where $g\left(x^{a}\right)$ is analytic defines an analytic change of coordinates on the elementary region for which $x^{3 \prime}$ is a new periodic coordinate (with period $2 \pi$ ) along the null generators. Furthermore, by construction, the new gaussian null coordinate system generated by this new chart on the elementary region leads to the desired condition $\dot{\phi}_{t^{\prime}}^{\prime}=k=$ constant. The above argument was given for $k>0$, but the same solution applies equally well for $k<0$. In fact, these two cases are essentially equivalent since a coordinate transformation of the type $t \rightarrow-t, x^{3} \rightarrow-x^{3}, x^{a} \rightarrow x^{a}$ preserves the gaussian null form of the metric but changes the sign of $\phi_{, t}$.

For the degenerate case we set $k=0$ in Eq. (3.11) and solve directly to get

$$
u\left(x^{3}, x^{a}\right)=\frac{\partial h\left(x^{3}, x^{a}\right)}{\partial x^{3}}=\frac{2 \pi p\left(x^{3}, x^{a}\right)}{\int_{0}^{2 \pi} d y\left(p\left(y, x^{a}\right)\right)},
$$

where $p\left(x^{3}, x^{a}\right)$ is defined by Eq. (3.12) as before. It is straightforward to verify that this solution has all the properties (i)-(ii) given above for the non-degenerate case. Thus, any coordinate transformation of the form

$$
x^{3^{\prime}}=g\left(x^{a}\right)+\frac{2 \pi \int_{0}^{x^{3}} d z\left(p\left(z, x^{a}\right)\right)}{\int_{0}^{2 \pi} d y\left(p\left(y, x^{a}\right)\right)}, \quad x^{a^{\prime}}=x^{a},
$$

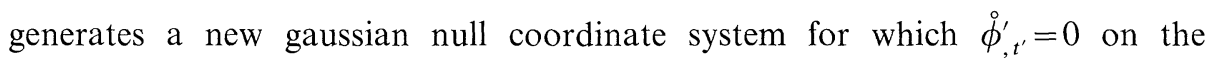
elementary region.

Some insight into the significance of the new coordinate $x^{3^{\prime}}$ can be gained by studying the geodesic equations for the null generators of $N$. Recalling Eqs. (3.9) we see that they now reduce to

$$
\frac{d^{2} x^{3^{\prime}}}{d \lambda^{2}}-\frac{k}{2}\left(\frac{d x^{3^{\prime}}}{d \lambda}\right)^{2}=0
$$

where $\lambda$ is an affine parameter. Solving this explicitly we get, for $k \neq 0$,

$$
x^{3^{\prime}}(\lambda)=x^{3^{\prime}}(0)-\frac{2}{k} \ln \left[1-\frac{k}{2} \lambda \frac{d x^{3^{\prime}}(0)}{d \lambda}\right]
$$

and, for $k=0$,

$$
x^{3^{\prime}}(\lambda)=x^{3^{\prime}}(0)+\lambda \frac{d x^{3^{\prime}}(0)}{d \lambda} .
$$

Thus in the non-degenerate case the null generators are complete in only one direction, whereas in the degenerate case they are complete in both directions. Note also that when ${\stackrel{\phi}{, t^{\prime}}}^{\prime}=k>0(k<0)$ the closed integral curves of $\frac{\partial}{\partial x^{3^{\prime}}}$ become closed timelike curves for small negative (positive) values of $t^{\prime}$, since $g_{3^{\prime} 3^{\prime}}=\phi^{\prime}$ is negative in that region. This accords with a result on compact Cauchy horizons given by Hawking and Ellis [7]. 
Returning to the main stream of the argument we now have, in the new gaussian null coordinates, $\mu_{a b, 3^{\prime}}^{\prime}=0$ (since this holds in any such coordinate system) ${\stackrel{\circ}{\phi}, t^{\prime}}^{\prime}=k=$ constant and thus, from the analogue of Eq. (3.2) in the new coordinate system, $\hat{\beta}_{b, t^{\prime} 3^{\prime}}^{\prime}=0$. Henceforth, to simplify the notation, we shall delete the primes on the new coordinates and metric functions.

With this notational change we now consider the equation $R_{a b}=0$ restricted to the null surface $t=0$ and reduced through the use of $\dot{\phi}_{, t}=k=$ constant, $\dot{\mu}_{a b, 3}=0$ and $\stackrel{\circ}{b, t 3}_{b}=0$. The result is

$0=\left.\left\{-\left(\mu_{a b, t}\right)_{, 3}+\frac{\phi_{, t}}{2} \mu_{a b, t}+\frac{1}{2}\left(\beta_{a, t b}+\beta_{b, t a}-2 \beta_{c, t}{ }^{(2)} \Gamma_{a b}^{c}\right)+{ }^{(2)} R_{a b}-\frac{1}{2} \beta_{a, t} \beta_{b, t}\right\}\right|_{t=0}$.

Differentiating this equation with respect to $x^{3}$ and using the invariance of $\dot{\phi}_{, t}, \stackrel{\beta}{a, t}_{a}$, and $\stackrel{\circ}{\mu}_{a b}$ with respect to $\frac{\partial}{\partial x^{3}}$ we get

$$
0=-\left(\stackrel{\circ}{\mu}_{a b, t}\right)_{, 33}+\frac{k}{2}\left(\stackrel{\circ}{\mu}_{a b, t, 3}\right)_{,}
$$

Integrating explicitly and demanding smoothness on the closed null orbits (or appealing to the maximum principle) leads to $\left(\dot{\mu}_{a b, t}\right)_{3}=0$.

To summarize, we have now derived the existence of a new gaussian null coordinate system on a neighborhood of the original elementary region for which the transformed metric functions satisfy

$$
\begin{gathered}
\stackrel{\circ}{\phi}=\stackrel{\circ}{\beta}_{a}=\stackrel{\leftrightarrow}{\mu}_{a b, 3}=0, \\
\stackrel{\circ}{\phi}, t=k=\text { const, } \quad\left(\stackrel{\circ}{\beta}_{a, t}\right)_{, 3}=0, \\
\left(\stackrel{\mu}{\mu}_{a b, t}\right)_{, 3}=0 .
\end{gathered}
$$

Thus all the metric functions and their first time derivatives are independent of $x^{3}$ on the initial surface $t=0$.

We can now proceed inductively to show that all higher time derivatives of the metric functions $\phi, \beta_{a}, \mu_{a b}$ are independent of $x^{3}$ at $t=0$, and thus that any analytic solution of the field equations has $\frac{\partial}{\partial x^{3}}$ as a (locally defined) Killing vector field throughout the gaussian null coordinate region considered.

Assume, as an inductive hypothesis, that $\phi, \beta_{a}, \mu_{a b}$ and all their time derivatives up through order $n$ (with $n \geqq 1$ ) are independent of $x^{3}$ at $t=0$, i.e. that

$$
0=\left(\left.\frac{\partial^{k} \phi}{\partial t^{k}}\right|_{t=0}\right)_{, 3}=\left(\left.\frac{\partial^{k} \beta_{a}}{\partial t^{k}}\right|_{t=0}\right)_{, 3}=\left(\left.\frac{\partial^{k} \mu_{a b}}{\partial t^{k}}\right|_{t=0}\right)_{, 3}
$$

for all $k$ such that $0 \leqq k \leqq n$. Differentiate the equation $R_{t 3}=0, n-1$ times with respect to $t$, and set $t=0$ to derive an expression for $\left.\left(\frac{\partial^{n+1}}{\partial t^{n+1}} \phi\right)\right|_{t=0}$ in terms of $x^{3}$-invariant quantities. Differentiate the equation $R_{t b}=0, n-1$ times with respect to $t$, and set $t=0$ to derive an expression for $\left.\left(\frac{\partial^{n+1}}{\partial t^{n+1}} \beta_{b}\right)\right|_{t=0}$ in terms of $x^{3}$-invariant 
quantities. Now, differentiate the equation $R_{a b}=0, n$ times with respect to $t$, set $t=0$ and use the above results for $\left.\left(\frac{\partial^{n+1}}{\partial t^{n+1}} \phi\right)\right|_{t=0} ^{a b}$ and $\left.\left(\frac{\partial^{n+1}}{\partial t^{n+1}} \beta_{b}\right)\right|_{t=0}$, together with those of Eq. (3.27) to derive an equation of the form

$$
\begin{aligned}
0= & \left.\frac{\partial^{n}}{\partial t^{n}} R_{a b}\right|_{t=0}=-\left(\left.\frac{\partial^{n+1}}{\partial t^{n+1}} \mu_{a b}\right|_{t=0}\right)_{, 3}+\left.\left(\begin{array}{c}
\text { positive } \\
\text { constant }
\end{array}\right) \frac{\stackrel{\phi}{\phi}_{, t}}{2}\left(\frac{\partial^{n+1}}{\partial t^{n+1}} \mu_{a b}\right)\right|_{t=0} \\
& +\left\{\text { terms independent of } x^{3}\right\} .
\end{aligned}
$$

Differentiate Eq. (3.29) with respect to $x^{3}$ and apply the maximum principle to the resulting equation for $\left.\left(\frac{\partial^{n+1}}{\partial t^{n+1}} \mu_{a b}\right)\right|_{t=0}$ to show that $\left.\left(\frac{\partial^{n+1}}{\partial t^{n+1}} \mu_{a b}\right)\right|_{t=0}$ is independent of $x^{3}$. Thus $\phi, \beta_{a}, \mu_{a b}$ and their time derivatives up through order $n+1$ are all independent of $x^{3}$ at $t=0$. This completes the inductive argument and shows that any analytic vacuum solution is independent of $x^{3}$ in the specially constructed gaussian null coordinate chart introduced above.

Notice that in the non-degenerate case $\left(\dot{\phi}_{, t}=k \neq 0\right)$, one can now solve Eq. (3.29) for $\left.\left(\frac{\partial^{n+1}}{\partial t^{n+1}} \mu_{a b}\right)\right|_{t=0}$ in terms of $x^{3}$-invariant quantities whereas, in the degenerate case, Eq. (3.29) does not determine this quantity. Thus in the nondegenerate case we can systematically recover the entire power series expansions for the metric functions from their values and that of their first-time derivatives at $t=0$ whereas, in the degenerate case, we cannot in general do so.

The above argument may be readily generalized to encompass electrovacuum solutions. To see this one first applies the maximum principle argument to the equation $\left.\left(R_{33}-8 \pi T_{33}\right)\right|_{t=0}=0$ to show that

$$
\stackrel{\circ}{\mu}_{a b, 3}=\stackrel{\circ}{F}_{3 a}=0 \text {. }
$$

Next, one restricts the Maxwell equation ${ }^{(4)} \nabla_{\beta} F^{t \beta}=0$ to $t=0$ and uses (3.30) to derive $\stackrel{\circ}{F}_{3 t, 3}=0$. Combining these results with the equation $\left.F_{[a b, 3]}\right|_{t=0}=0$ yields $\stackrel{\circ}{F}_{a b, 3}=0$. In view of these results, we find that $\left.T_{3 b}\right|_{t=0}=0$ and thus that Eq. (3.2) holds without modification. We can, therefore, repeat the argument which transforms the metric to new gaussian null coordinates in which $\dot{\phi}_{, t}=k=$ constant and $\AA_{b, t 3}=0$ without disturbing the foregoing results. In the new coordinates we find immediately that $\left.T_{a b, 3}\right|_{t=0}=0$. Therefore, differentiating the equation $\left.\left(R_{a b}-8 \pi T_{a b}\right)\right|_{t=0}=0$ with respect to $x^{3}$ leads back, without modification, to Eq. (3.26) and thus to $\left(\dot{\mu}_{a b, t}\right)_{3}=0$. Finally, from the Maxwell equations $\left.{ }^{(4)} \nabla_{\beta} F^{a \beta}\right|_{t=0}=0$ and $\left.F_{[a 3, t]}\right|_{t=0}=0$ we derive, after differentiation with respect to $x^{3}$,

$$
\left.\left(-\phi_{, t} F_{c t, 3}+2 F_{c t, 33}\right)\right|_{t=0}=0 .
$$

Recalling that $\dot{\phi}_{, t}=$ constant and integrating this explicitly (or using the maximum principle), we get $\stackrel{\circ}{F}_{c t, 3}=0$.

To summarize, we have shown that, in a suitably chosen gaussian null coordinate system, the initial data satisfy

$$
\begin{aligned}
\stackrel{\circ}{\phi} & =\stackrel{\circ}{\beta}_{a}=\stackrel{\circ}{\mu}_{a b, 3}=\stackrel{\circ}{F}_{3 a}=\stackrel{\circ}{F}_{a t, 3}=\stackrel{\circ}{F}_{t 3,3}=\stackrel{\circ}{F}_{a b, 3}=\stackrel{\circ}{\beta}_{a, t 3} \\
& =\stackrel{\circ}{\mu}_{a b, t 3}=0, \quad \stackrel{\circ}{\phi}, t_{,}=\text {constant },
\end{aligned}
$$


and thus are all independent of $x^{3}$. We can now repeat the inductive argument to show that all the successive higher time derivatives of the field variables are independent of $x^{3}$ at the initial surface $t=0$. To see this suppose that, for any $n \geqq 1$, we have, in addition to (3.22),

$$
\begin{aligned}
0=\left(\left.\frac{\partial^{k} \phi}{\partial t^{k}}\right|_{t=0}\right)_{, 3} & =\left(\left.\frac{\partial^{k} \beta_{a}}{\partial t^{k}}\right|_{t=0}\right)_{, 3}=\left(\left.\frac{\partial^{k} \mu_{a b}}{\partial t^{k}}\right|_{t=0}\right)_{, 3}, \\
0 & =\left(\left.\frac{\partial^{l} F_{\mu v}}{\partial t^{l}}\right|_{t=0}\right)_{, 3},
\end{aligned}
$$

for all $k$ and $l$ such that $0 \leqq k \leqq n, 0 \leqq l \leqq n-1$. Taking $n-1$ time derivatives of the equations, $\left(R_{t 3}-8 \pi T_{t 3}\right)=0,\left(R_{t a}-8 \pi T_{t a}\right)=0, F_{[a b, t]}=0$, and $F_{[a 3, t]}=0$, and setting $t=0$, we find immediately that $\left.\left(\frac{\partial^{n+1}}{\partial t^{n+1}} \phi\right)\right|_{t=0},\left.\left(\frac{\partial^{n+1}}{\partial t^{n+1}} \beta_{a}\right)\right|_{t=0},\left.\left(\frac{\partial^{n}}{\partial t^{n}} F_{a b}\right)\right|_{t=0}$, and $\left.\left(\frac{\partial^{n}}{\partial t^{n}} F_{a 3}\right)\right|_{t=0}$ are all independent of $x^{3}$. Taking $n-1$ time derivatives of the equation ${ }^{(4)} \nabla_{\beta} F^{3 \beta}=0$ and setting $t=0$ then shows that $\left.\left(\frac{\partial^{n}}{\partial t^{n}} F_{t 3}\right)\right|_{t=0}$ is independent of $x^{3}$.

Using these results we next compute the $n^{\text {th }}$ time derivative of the equation $\left(R_{a b}-8 \pi T_{a b}\right)=0$, set $t=0$ and differentiate the resultant expression with respect to $x^{3}$. The source term $\left.\left(\frac{\partial^{n+1}}{\partial t^{n} \partial x^{3}} T_{a b}\right)\right|_{t=0}$ drops out by virtue of the invariance results derived above and the fact that the coefficients of the quantity $\left.\left(\frac{\partial^{n}}{\partial t^{n}} F_{t a}\right)\right|_{t=0}$, which has not yet been proven invariant, vanish at $t=0$. Therefore, we can repeat the maximum principle argument given in the vacuum case to show that $\left.\left(\frac{\partial^{n+1}}{\partial t^{n+1}} \mu_{a b}\right)\right|_{t=0}$ is independent of $x^{3}$.

Finally we compute the $n^{\text {th }}$ time derivative of the equation ${ }^{(4)} \nabla_{\beta} F^{a \beta}=0$, use the $n^{\text {th }}$ time derivative of $F_{[a 3, t]}=0$ to reexpress $\left(\frac{\partial^{n+1}}{\partial t^{n+1}} F_{a 3}\right)$, and thus derive, at $t=0$, an equation of the form

$$
\begin{aligned}
& \left(\left.\frac{\partial^{n}}{\partial t^{n}} F_{a t}\right|_{t=0}\right),\left.\left(\begin{array}{c}
\text { positive } \\
\text { constant }
\end{array}\right) \dot{\phi}_{, t}\left(\frac{\partial^{n} F_{a t}}{\partial t^{n}}\right)\right|_{t=0} \\
& =\left\{\text { quantities independent of } x^{3}\right\} .
\end{aligned}
$$

Differentiating this with respect to $x^{3}$ and applying the maximum principle argument, we find that $\left.\left(\frac{\partial^{n}}{\partial t^{n}} F_{a t}\right)\right|_{t=0}$ is independent of $x^{3}$.

This completes the inductive argument in the Einstein Maxwell case. Note that if $\stackrel{\circ}{\phi}, t_{0}=k \neq 0$, then all higher order time derivatives are determined in terms of $\stackrel{\circ}{F}_{\mu \nu}$, $\stackrel{\circ}{\mu}_{a b}, \stackrel{\circ}{\phi}, t_{i} \stackrel{\circ}{a, t}_{\text {t }}$, and $\stackrel{\circ}{\mu}_{a b, t}$. Thus the power series expansions of $g$ and $F$ can be uniquely recovered from this initial data in the non-degenerate case. 


\section{B. Global Arguments}

The above results show that, in each suitably constructed gaussian null coordinate system $\left(t, x^{3}, x^{a}\right)$, the spacetime metric $g$ and Maxwell field $F$ are invariant with respect to the (locally defined) vector field $\frac{\partial}{\partial x^{3}}$. We wish to show that these locally defined vector fields patch together naturally to give an analytic (Killing) vector field on a full neighborhood of the null surface $N$. Suppose that $\left(t, x^{3}, x^{a}\right)$ and $\left(\tilde{t}, \tilde{x}^{3}, \tilde{x}^{a}\right)$ are any two such coordinate systems whose elementary regions overlap in some open subset $N$ of the null surface $N$. Then these charts will overlap on some neighborhood of $N_{n}$ in ${ }^{(4)} V$, and we wish to show that the two locally defined Killing fields $Y=\frac{\partial}{\partial x^{3}}$ and $\tilde{Y}=\frac{\partial}{\partial \tilde{x}^{3}}$ coincide on their common domain of definition (i.e., that their components satisfy the covariance relation $Y^{\alpha}=\frac{\partial x^{\alpha}}{\partial \tilde{x}^{\beta}} \tilde{Y}^{\beta}$ ). To accomplish this we shall show that $Y$ and $\tilde{Y}$ coincide when restricted to $N_{\cap}$, and then appeal to the uniqueness of solutions of Killing equations to show that they coincide throughout their common domain of definition.

By construction the two coordinate systems have $\left.\phi_{, t}\right|_{t=0}=k$ and $\left.\tilde{\phi}_{, \hat{i}}\right|_{\tilde{t}=0}=\tilde{k}$ for some constants $k$ and $\tilde{k}$. We may assume that $\left.\frac{\partial \tilde{x}^{3}}{\partial x^{3}}\right|_{t=0}>0$, since otherwise we could achieve this by the transformation $\tilde{t} \rightarrow-\tilde{t}, \tilde{x}^{3} \rightarrow-\tilde{x}^{3}, \tilde{x}^{a} \rightarrow \tilde{x}^{a}$. By relating the two coordinates $x^{3}$ and $\tilde{x}^{3}$ to an affine parameter along each null geodesic generator lying in $N_{\cap}$, we shall show that $k=\tilde{k}$ and that $\left.\frac{\partial \tilde{x}^{3}}{\partial x^{3}}\right|_{N_{n}}=1$. This in turn will establish the desired equivalence of $\left.Y\right|_{N_{n}}$ and $\left.\tilde{Y}\right|_{N_{n}}$.

Let $\gamma$ be an affinely parametrized null generator lying in $N_{n}$ (with affine parameter $\lambda$ ). Then $t(\lambda)=\tilde{t}(\lambda)=0, x^{a}(\lambda)$, and $\tilde{x}^{a}(\lambda)$ are constant and, from Eq. (3.22),

$$
0=\frac{d^{2} x^{3}}{d \lambda^{2}}-\frac{k}{2}\left(\frac{d x^{3}}{d \lambda}\right)^{2}=\frac{d^{2} \tilde{x}^{3}}{d \lambda^{2}}-\frac{\tilde{k}}{2}\left(\frac{d \tilde{x}^{3}}{d \lambda}\right)^{2}
$$

If $\gamma$ is complete in only one direction then, since $\left.\frac{\partial \tilde{x}^{3}}{\partial x^{3}}\right|_{N_{n}}>0, k$, and $\tilde{k}$ are either both positive or both negative. If $\gamma$ is complete in both directions, then $k=\tilde{k}=0$.

Consider first the non-degenerate case for which we have the solutions

$$
\begin{aligned}
& x^{3}(\lambda)=x^{3}(0)-\frac{2}{k} \ln \left[1-\frac{k}{2} \lambda \frac{d x^{3}(0)}{d \lambda}\right], \\
& \tilde{x}^{3}(\lambda)=\tilde{x}^{3}(0)-\frac{2}{\tilde{k}} \ln \left[1-\frac{\tilde{k}}{2} \lambda \frac{d \tilde{x}^{3}(0)}{d \lambda}\right],
\end{aligned}
$$

and the covariance relation

$$
\frac{d \tilde{x}^{3}(0)}{d \lambda}=\left.\frac{\partial \tilde{x}^{3}}{\partial x^{3}}\right|_{x^{\mu}(0)} \frac{d x^{3}(0)}{d \lambda}
$$


Take $\frac{d x^{3}(0)}{d \lambda}>0$ for definiteness and let $\lambda_{n}$ be the value of $\lambda$ after $n$ full circuits so that

$$
\begin{aligned}
& x^{3}\left(\lambda_{n}\right)=x^{3}(0)+2 \pi n, \\
& \tilde{x}^{3}\left(\lambda_{n}\right)=\tilde{x}^{3}(0)+2 \pi n,
\end{aligned}
$$

and allow $n$ to be either positive or negative. Equations (3.36) and (3.38) give

$$
\begin{aligned}
& 1-\frac{k}{2} \lambda_{n} \frac{d x^{3}(0)}{d \lambda}=e^{-n \pi k}, \\
& 1-\frac{\tilde{k}}{2} \lambda_{n} \frac{d \tilde{x}^{3}(0)}{d \lambda}=e^{-n \pi \tilde{k}} .
\end{aligned}
$$

Combining these equations with the covariance relation (3.37) gives, for all integral $n \neq 0$,

$$
\left.\frac{\partial \tilde{x}^{3}}{\partial x^{3}}\right|_{x^{\mu}(0)}=\frac{k}{\tilde{k}} \frac{\left(1-e^{-n \pi \tilde{k}}\right)}{\left(1-e^{-n \pi k}\right)}
$$

However, the left-hand side of this equation is independent of $n$, whereas the righthand side is independent of the initial condition $x^{\mu}(0)$, which can be chosen arbitrarily within $N_{\cap}$. Equality is possible only when $k=\tilde{k}$ and $\left.\frac{\partial \tilde{x}^{3}}{\partial x^{3}}\right|_{N_{n}}=1$.

It follows that the two overlapping systems of coordinates are related at $t=\tilde{t}=0$ by a transformation of the form

$$
\tilde{x}^{3}=x^{3}+\sigma\left(x^{a}\right), \quad \tilde{x}^{a}=f^{a}\left(x^{b}\right), \quad \text { at } \quad t=\tilde{t}=0,
$$

where $f^{a}\left(x^{b}\right)$ and $\sigma\left(x^{a}\right)$ are analytic and $\operatorname{det}\left(\frac{\partial f^{a}}{\partial x^{b}}\right) \neq 0$. Using this result to reexpress the vector field $\left.\frac{\partial}{\partial x^{3}}\right|_{t=0}$ in terms of the coordinates $\left(\tilde{t}, \tilde{x}^{3}, \tilde{x}^{a}\right)$, we get

$$
\left.\frac{\partial}{\partial x^{3}}\right|_{t=0}=\left.\left(\frac{\partial \tilde{x}^{\alpha}}{\partial x^{3}} \frac{\partial}{\partial \tilde{x}^{\alpha}}\right)\right|_{t=0}=\left.\frac{\partial}{\partial \tilde{x}^{3}}\right|_{\tilde{t}=0},
$$

which establishes the equality $\left.Y\right|_{N_{\cap}}=\left.\tilde{Y}\right|_{N \cap}$. A completely analogous argument applies in the degenerate case $k=\tilde{k}=0$.

In their common domain of definition $Y$ and $\tilde{Y}$ both satisfy Killing's equation and restrict to a common vector field on $N_{n}$. It follows that $X=\tilde{Y}-Y$ is a Killing field defined on this domain which vanishes on $N_{\cap}$. However, this implies that $X$ vanishes throughout its domain of definition, since the Killing equations

$$
X_{\mu, t}+X_{t, \mu}-2^{(4)} \Gamma_{\mu t}^{v} X_{v}=0
$$

determine $X$ uniquely from data $\left.X\right|_{t=0}$ and have only the trivial solution $X=0$ if $\left.X\right|_{t=0}=0$.

Let $Y$ now designate the Killing field which has thus been shown to exist on a neighborhood of $N$ in $\left({ }^{(4)} V, g\right)$. Since the quantity $k$, defined originally only locally, 
has now been shown to have a constant value over $N$, we can meaningfully speak of degenerate versus non-degenerate null surfaces. The non-degenerate null surfaces are always Cauchy horizons as we shall now show.

Define a real analytic function $\zeta$ by

$$
\zeta=Y \cdot Y=g_{\mu \nu} Y^{\mu} Y^{\nu},
$$

and note that, in any gaussian null coordinate chart,

$$
\zeta=g_{33}=\phi .
$$

Since $\left.\left(\partial_{\mu} \zeta\right)\right|_{t=0}=(k, 0,0,0)$ and since $k \neq 0$, by assumption $\zeta$ has no critical points in some neighborhood of $N$ and has $N$ as a level surface corresponding to the value $\zeta=0$. It follows that the surfaces $\zeta=$ constant are, for sufficiently small $|\zeta|$, diffeomorphic to $N$ and foliate a neighborhood of $N$ in ${ }^{(4)} V$. One proves this by introducing a Riemannian metric $\tilde{g}$ on a neighborhood of $N$ and dragging $N$ along the flow of $Z^{\mu}=\left(\frac{\tilde{g}^{\mu \nu} \partial_{\nu} \zeta}{\tilde{g}^{\alpha \beta}\left(\partial_{\alpha} \zeta\right)\left(\partial_{\beta} \zeta\right)}\right)$ to generate the nearby $\zeta=$ constant surfaces.

Suppose for definiteness that $k>0$ and thus, from a straightforward computation, that

$$
\begin{aligned}
\left.g^{\mu \nu}\left(\partial_{\mu} \zeta\right)\left(\partial_{\nu} \zeta\right)\right|_{t}=0 & =0, \\
{\left.\left[\frac{\partial}{\partial t}\left(g^{\mu \nu}\left(\partial_{\mu} \zeta\right)\left(\partial_{\nu} \zeta\right)\right)\right]\right|_{t=0} } & =-k^{3}<0 .
\end{aligned}
$$

It follows that $\partial_{\mu} \zeta$ is timelike for all sufficiently small $t>0$ and thus, since $\left.\frac{\partial \zeta}{\partial t}\right|_{t=0}=k>0$, for all sufficiently small $\zeta>0$. In other words, for some constant $\zeta_{0}>0$, the level surfaces $\zeta=$ constant $\in\left(0, \zeta_{0}\right)$ are all spacelike.

Introduce $\zeta$ as a new time coordinate on the region of $\left({ }^{(4)} \mathrm{V}, g\right)$ foliated by the level surfaces of $\zeta$ with values in the range $\left(0, \zeta_{0}\right)$, and write the metric as

$$
g={ }^{(3)} g_{i j}\left(d x^{i}+N^{i} d \zeta\right)\left(d x^{j}+N^{j} d \zeta\right)-N^{2} d \zeta^{2},
$$

where ${ }^{(3)} g_{i j}$ is Riemannian on each $\zeta=$ constant surface, and where $N>0$. Let $a$ and $b$ be any two constants such that $a<b$ and $[a, b] c\left(0, \zeta_{0}\right)$, and consider the compact subset of spacetime foliated by the surfaces $\zeta=$ constant $\in[a, b]$. Let $\gamma$ be any inextendible timelike curve in this region parameterized by proper time $s$, so that

$$
N^{2}\left(\frac{d \zeta}{d s}\right)^{2}=1+{ }^{(3)} g_{i j}\left(\frac{d x^{i}}{d s}+N^{i} \frac{d \zeta}{d s}\right)\left(\frac{d x^{j}}{d s}+N^{j} \frac{d \zeta}{d s}\right)
$$

Since ${ }^{(3)} g_{i j}$ is Riemannian and $N$ is bounded (and bounded away from zero) on this compact region, we see that $\left(\frac{d \zeta}{d s}\right)$ is bounded away from zero, and thus that $\gamma$ cannot avoid intersecting each surface $\zeta=$ constant $\in[a, b]$ precisely once. Since this holds for any $[a, b] \subset\left(0, \zeta_{0}\right)$, it follows that the spacetime foliated by the surfaces $\zeta=$ constant $\epsilon\left(0, \zeta_{0}\right)$ is globally hyperbolic and has these surfaces as 
Cauchy hypersurfaces. Since the surface $\zeta=0$ lies at the boundary of the globally hyperbolic region and is null, it is necessarily a Cauchy horizon.

We have thus proven:

Theorem 1. Every analytic vacuum or electrovacuum spacetime $\left({ }^{(4)} V, g\right)$ with a compact null hypersurface $N$ ruled by closed null generators in the sense of Sect. II has an analytic Killing field $Y$ defined on some neighborhood of $N$ in ${ }^{(4)} V$, and $Y$ has closed integral curves in this neighborhood. Furthermore, $\left.Y\right|_{N}$ is null and thus tangent to the generators of $N$. In the non-degenerate case (characterized by $\left.\left.\left(\partial_{\mu}(Y \cdot Y)\right)\right|_{N} \neq 0\right) N$ is a Cauchy horizon, the region $\zeta=Y \cdot Y>0$ is, for sufficiently small $\zeta$, globally hyperbolic and the region $\zeta<0$ has (for $|\zeta|$ sufficiently small) closed timelike curves through every point. If Maxwell fields are present, then the field tensor $F$ is invariant with respect to $Y$ (i.e., $\mathscr{L}_{Y} F=0$ ).

We remark that the closure of the integral curves of $Y$ follows from its construction locally as the vector field $\frac{\partial}{\partial x^{3}}$ of a gaussian null coordinate chart. These closed curves are timelike in the region $\zeta<0$ since $Y \cdot Y=\zeta$ is negative in this region.

One can actually extend the Killing field defined near $N$ to a Killing field defined on the maximal Cauchy development of the globally hyperbolic region described above. The techniques for doing this are discussed (for the vacuum case) by Fischer et al. in [8]. One propagates $Y$ to define a vector field on the maximal Cauchy development by solving a certain second order, linear hyperbolic equation. Next one shows that $h \equiv \mathscr{L}_{\mathrm{Y}} g$ satisfies another second order linear hyperbolic equation and that $h$ vanishes everywhere provided its Cauchy data vanishes. In our case, the Cauchy data vanishes since $h$ vanishes near $N$. A similar argument can be given for the Einstein-Maxwell equations.

It is worth noting that the Killing field $Y$ satisfies the equation

$$
\left.Y^{\beta(4)} \nabla_{\beta} Y^{\alpha}\right|_{t=0}=-\left.\frac{k}{2} Y^{\alpha}\right|_{t=0},
$$

which shows that the constant $\left(-\frac{k}{2}\right)$ is the analogue, for cosmological Cauchy horizons, of the surface gravity defined for stationary black hole event horizons [9].

\section{Degenerate and Non-degenerate Solutions on $T^{3} \times R$}

In this section we specialize the spacetime manifold to ${ }^{(4)} V=\mathbf{T}^{3} \times \mathbf{R}$ and take $\left\{x^{i}\right\}$ $=\left\{x^{3}, x^{a}\right\}$ to be periodic coordinates (with period $2 \pi$ ) on the three-torus. As in the conclusion of the previous section, we find $\frac{\partial}{\partial x^{3}}$ to be a Killing field of $\left({ }^{(4)} V, g\right)$ with closed null orbits on the surface $t=0$. For this case we shall be able to characterize the degenerate and non-degenerate vacuum solutions completely. Presumably one could treat the electrovacuum case with the same techniques but, except for 
displaying a class of degenerate electrovacuum solutions, we shall not pursue that question here.

It is easy to guess a large family of degenerate solutions on $\mathbf{T}^{3} \times \mathbf{R}$. The plane wave metrics

$$
d s^{2}=2 d t d x^{3}+\mu_{a b}(t) d x^{a} d x^{b}
$$

satisfy the vacuum Einstein equations, provided $\mu_{a b}(t)$ satisfies

$$
R_{t t}=-\frac{1}{2} \mu^{a b} \mu_{a b, t t}+\frac{1}{4} \mu^{a c} \mu^{b d} \mu_{a b, t} \mu_{c d, t}=0 .
$$

These are clearly degenerate since $\phi=g_{33}$ vanishes everywhere. One gets a class of degenerate Einstein-Maxwell solutions by taking $F_{t 3}=F_{a b}=F_{a 3}=0$, and replacing Eq. (4.2) by

$$
R_{t t}=2 F_{t a} F_{t b} \mu^{a b}
$$

In these solutions $F_{t a}(t)$ and the "conformal part" $\left(\frac{\mu_{a b}}{\sqrt{\mu}}\right)$ of $\mu_{a b}(t)$ may be specified arbitrarily as functions of $t$ with $\sqrt{\mu}$ then determined by Eqs. (4.2) and (4.3). Since $\phi \equiv 0$ these solutions have a compact null surface $N_{t} \approx \mathbf{T}^{3}$ through every spacetime event.

In this section we shall prove that every degenerate vacuum solution on $\mathbf{T}^{3} \times \mathbf{R}$ with simply periodic null orbits (i.e., with null generators tangent to a periodic coordinate vector field $\left.\frac{\partial}{\partial x^{3}}\right)$ is diffeomorphic to a plane wave solution. Presumably a similar result holds for the electrovacuum case but we shall not attempt to prove it.

In general a degenerate solution has, in appropriate coordinates, $\stackrel{\phi}{, t}_{t}=0$ and $g_{\mu v, 3}=0$. The equation $\stackrel{\circ}{R}_{a b}=0$ thus reduces to

$$
\left.\left\{\frac{1}{2}\left(\beta_{a, t \mid b}+\beta_{b, t \mid a}\right)+{ }^{(2)} R_{a b}-\frac{1}{2} \beta_{a, t} \beta_{b, t}\right\}\right|_{t=0}=0,
$$

where we have adopted the notation $\mid a$ for ${ }^{(2)} \nabla_{a}$. Taking the trace of this equation, integrating over any $x^{3}=$ constant two-torus and appealing to the Gauss-Bonnet theorem, we get

$$
\left.\beta_{a, t}\right|_{t=0}=\left.{ }^{(2)} R_{a b}\right|_{t=0}=0 .
$$

Thus $\stackrel{\circ}{\mu}_{a b}$ is flat. It follows immediately from the $\stackrel{\circ}{R}_{t 3}=0$ equation that $\left.\phi_{, t t}\right|_{t=0}=0$. Next we consider the equation $\left.\frac{\partial R_{a b}}{\partial t}\right|_{t=0}=0$ and note that, since ${ }^{(2)} R_{a b}$ $=\frac{1}{2} \mu_{a b}{ }^{(2)} R$ and $\stackrel{\circ}{\mu}_{a b}$ is flat, we have

This leads to

$$
\left.\frac{\partial^{(2)} R_{a b}}{\partial t}\right|_{t=0}=\left.\frac{1}{2} \stackrel{\mu}{a b}_{a b}\left(\frac{\partial}{\partial t}{ }^{(2)} R\right)\right|_{t=0} .
$$

$$
\left.\left\{\beta_{a, t t \mid b}+\beta_{b, t t \mid a}+\mu_{a b} \frac{\partial}{\partial t}{ }^{(2)} R\right\}\right|_{t=0}=0
$$


which shows that $\left.\beta_{a, t t}\right|_{t=0}$ is a conformal Killing field of $\stackrel{\circ}{a b}_{a b}$. However, this implies that $\left.\left(\beta_{a, t t \mid b}\right)\right|_{t=0}=0$, as follows from taking the divergence of the conformal Killing equation and using the flatness of $\stackrel{\circ}{a b}_{a b}$. Thus we now get

$$
\left.\left(\beta_{a, t t \mid b}\right)\right|_{t=0}=\left.\left(\frac{\partial}{\partial t}^{(2)} R_{a b}\right)\right|_{t=0}=0 .
$$

Furthermore, the $\stackrel{\circ}{R}_{t b}=0$ equation reduces to

$$
\left.\left\{\beta_{b, t t}+\left(\mu^{a c} \mu_{a b, t}\right)_{\mid c}-\left(\mu^{a c} \mu_{a c, t}\right)_{\mid b}\right\}\right|_{t=0}=0 .
$$

Contracting this last equation with $\stackrel{\circ}{\mu}^{a b} \stackrel{\circ}{\beta}_{a, t t}$, integrating over $\mathbf{T}^{2}$, and appealing to the covariant constancy of $\AA_{a, t t}$, we get

$$
\left.\left(\beta_{a, t t}\right)\right|_{t=0}=\left.\left\{\left(\mu^{a c} \mu_{a b, t}\right)_{\mid c}-\left(\mu^{a c} \mu_{a c, t}\right)_{\mid b}\right\}\right|_{t=0}=0 .
$$

Thus $\stackrel{\circ}{\mu}_{a b, t}$ must satisfy this last equation as well as $\left.\frac{\partial^{(2)} R_{a b}}{\partial t}\right|_{t=0}=0$.

Using standard elliptic theory, we split $h_{a b} \equiv \stackrel{\circ}{\mu}_{a b, t}$ uniquely into $L^{2}$ orthogonal components

$$
h_{a b}=\stackrel{\circ}{\mu}_{a b, t}=h_{a b}^{\mathrm{tr}}+\left(V_{a \mid b}+V_{b \mid a}\right),
$$

where $h_{a b}^{\mathrm{tr} \mid b}=0$. Substituting this expression into Eq. (4.8) however, we find that $h_{a b}^{\mathrm{tr}}$ must be covariantly constant. Therefore, we write

$$
h_{a b}=h_{a b}^{c c}+V_{a \mid b}+V_{b \mid a},
$$

where $h_{a b \mid d}^{c c}=0$, and further split $V_{a}=V_{a}^{\mathrm{tr}}+\lambda_{\mid a}$, where $V_{a}^{\mathrm{tr} \mid a}=0$. Substituting this expression for $h_{a b}$ into Eq. (4.10) leads to $V_{b \mid a}^{\mathrm{tr}}=0$, so that $h_{a b}$ reduces to

$$
h_{a b}=\stackrel{\circ}{\mu}_{a b, t}=h_{a b}^{c c}+2 \lambda_{\mid a b},
$$

with $h_{a b \mid d}^{c c}=0$ and $\lambda$ arbitrary.

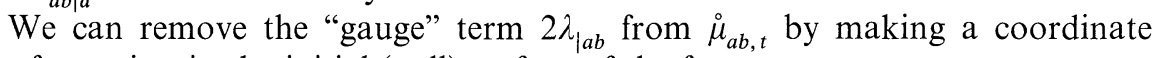
transformation in the initial (null) surface of the form

$$
x^{3^{\prime}}=x^{3}-\lambda\left(x^{a}\right), \quad x^{a^{\prime}}=x^{a},
$$

and generating (as discussed in Sect. IIB) the new gaussian null coordinate system determined thereby. Since $\left.\frac{\partial x^{3^{\prime}}}{\partial x^{3}}\right|_{t=0}=1$, this transformation will preserve the explicit symmetry (i.e., give $g_{\mu v, 3^{\prime}}^{\prime}=0$ ) and all the results derived above since the latter hold in any gaussian null coordinate system adapted to the symmetry. To establish the desired result, we need only evaluate $\left.\mu_{a b, t^{\prime}}^{\prime}\right|_{t^{\prime}=0}$ in terms of the original, unprimed metric quantities. A straightforward calculation gives

$$
\begin{gathered}
\left.\mu_{a b}^{\prime}\right|_{t^{\prime}=0}=\left.\mu_{a b}\right|_{t=0}, \\
\mu_{a b,\left.t^{\prime}\right|_{t^{\prime}=0} ^{\prime}}^{\prime}=\left.\mu_{a b, t}\right|_{t=0}-2 \lambda_{\mid a b}=h_{a b}^{c c} .
\end{gathered}
$$

We now work in the new coordinate system but, to simplify the notation, delete the primes on the new coordinates and metric functions. 
Summarizing the above results, we have shown that, after a suitable change of coordinates,

$$
\begin{gathered}
0=\stackrel{\circ}{\phi}^{\prime} \stackrel{\circ}{\phi}, t_{,}=\stackrel{\circ}{\phi}, t t=\stackrel{\circ}{\beta}_{a}=\stackrel{\circ}{\beta}_{a, t}=\stackrel{\circ}{\beta}_{a, t t}, \\
\stackrel{\circ}{\mu}_{a b} \text { is flat and } \stackrel{\circ}{\mu}_{a b, t \mid c}=0 .
\end{gathered}
$$

Assume as an inductive hypothesis that, for any $n \geqq 2$,

$$
\begin{aligned}
& \stackrel{\circ}{\phi}=\stackrel{\circ}{\phi}, t_{,}=\ldots=\frac{\partial^{n}}{\partial t^{n}} \stackrel{\circ}{\phi}=0 \text {, } \\
& \stackrel{\circ}{\beta}_{a}=\stackrel{\circ}{\beta}_{a, t}=\ldots=\frac{\partial^{n}}{\partial t^{n}} \stackrel{\circ}{\beta}_{a}=0 \text {, } \\
& \stackrel{\circ}{\mu}_{a b} \text { is flat and } \stackrel{\circ}{\mu}_{a b, t}, \ldots, \frac{\partial^{n-1}}{\partial t^{n-1}} \stackrel{\circ}{\mu}_{a b} \text { are all covariantly } \\
& \text { constant with respect to } \stackrel{\circ}{\mu}_{a b} \text {. }
\end{aligned}
$$

Taking $n-1$ time derivatives of $R_{t 3}=0$ gives $\left.\left(\frac{\partial^{n+1} \phi}{\partial t^{n+1}}\right)\right|_{t=0}=0$. Taking $n-1$ time derivatives of $R_{t b}=0$ leads to

$$
0=\left.\left\{\beta_{b}^{(n+1)}+\left(\mu^{a c} \mu_{a b}^{(n)}\right)_{\mid c}-\left(\mu^{a c} \mu_{a c}^{(n)}\right)_{\mid b}\right\}\right|_{t=0},
$$

where we have adopted the notation $\beta_{b}^{(n+1)}=\frac{\partial^{n+1}}{\partial t^{n+1}} \beta_{b}$, etc. and used the inductive hypothesis to simplify the result. In a similar way we get from the $n^{\text {th }}$ time derivative of $R_{a b}=0$ the equation

$$
0=\left.\left\{\left(\beta_{a}^{(n+1)}\right)_{\mid b}+\left(\beta_{b}^{(n+1)}\right)_{\mid a}+\mu_{a b} \frac{\partial^{n}}{\partial t^{n}}(2) R\right\}\right|_{t=0},
$$

where we have used the inductive hypothesis in various ways, in particular to show that

$$
{ }^{(2)} \stackrel{\circ}{R}_{a b}=\frac{\partial}{\partial t}{ }^{(2)} \stackrel{\circ}{R}_{a b}=\ldots=\frac{\partial^{n-1}}{\partial t^{n-1}} \stackrel{\circ}{R}_{a b}=0 \text {. }
$$

Arguing as before we get that

$$
\left.\left(\frac{\partial^{n+1}}{\partial t^{n+1}} \beta_{a}\right)\right|_{|b|}=0,\left.\quad \frac{\partial^{n}}{\partial t^{n}}{ }^{(2)} R_{a b}\right|_{t=0}=0 .
$$

In view of the inductive hypothesis, the latter equation reduces to the condition that the first variation of ${ }^{(2)} R_{a b}$ about $\stackrel{\circ}{\mu}_{a b}$ in a direction given by $\frac{\partial^{n}}{\partial t^{n}} \stackrel{\circ}{\mu}_{a b}$ is zero. As before, this leads to a splitting

$$
\stackrel{\circ}{\mu}_{a b}^{(n)}=\tilde{h}_{a b}^{c c}+2 \tilde{\lambda}_{\mid a b}+\tilde{V}_{a \mid b}^{\mathrm{tr}}+\tilde{V}_{b \mid a}^{\mathrm{tr}}, \quad \text { where } \quad \tilde{h}_{a b \mid d}^{c c}=0 \text {, and } \tilde{V}_{a}^{t r \mid a}=0 .
$$

Contracting Eq. (4.18) with $\stackrel{\circ}{\mu}^{a b} \stackrel{\circ}{\beta}_{a}^{(n+1)}$ and integrating over $\mathbf{T}^{2}$, we get [from covariant constancy of $\hat{\beta}_{a}^{(n+1)}$, cf., Eq. (4.21)], that 


$$
\left.\left(\frac{\partial^{n+1} \beta_{a}}{\partial t^{n+1}}\right)\right|_{t=0}=\left.\left\{\left(\mu^{a c} \mu_{a b}^{(n)}\right)_{\mid c}-\left(\mu^{a c} \mu_{a c}^{(n)}\right)_{\mid b}\right\}\right|_{t=0}=0 .
$$

Substituting the splitting (4.22) of $\dot{\mu}_{a b}^{(n)}$ into this last equation leads to $\tilde{V}_{a \mid b}^{\mathrm{tr}}=0$, and thus to

$$
\left.\frac{\partial^{n} \mu_{a b}}{\partial t^{n}}\right|_{t=0}=\tilde{h}_{a b}^{c c}+2 \tilde{\lambda}_{\mid a b} .
$$

Finally, taking $n-2$ time derivatives of $R_{t t}=0$ and using the above, we get

$$
\stackrel{\leftrightarrow}{\mu}^{a b} \tilde{h}_{a b}^{c c}+2 \tilde{\lambda}_{\mid a} \mid a=\text { constant }
$$

where the right-hand side is a complicated expression constructed from $\stackrel{\circ}{\mu}_{a b}, \stackrel{\circ}{\mu}_{a b, t}, \ldots, \stackrel{\circ}{\mu}_{a b}^{(n-1)}$. Since $\stackrel{\leftrightarrow}{\mu}^{a b} \tilde{h}_{a b}^{c c}$ is also constant, this equation implies that $\tilde{\lambda}=$ constant and thus that $\left.\left(\frac{\partial^{n} \mu_{a b}}{\partial t^{n}}\right)\right|_{t=0}$ is covariantly constant. One can always satisfy Eq. (4.25) by adjusting $\left(\dot{\mu}^{a b} \tilde{h}_{a b}^{c c}\right)$ appropriately. We have thus shown that

$$
\frac{\partial^{n} \stackrel{\circ}{\phi}}{\partial t^{n}}=\frac{\partial^{n}}{\partial t^{n}} \stackrel{\circ}{\beta}_{a}=\left(\frac{\partial^{n-1}}{\partial t^{n-1}} \stackrel{\leftrightarrow}{a b}_{a c}=0,\right.
$$

which completes the induction.

Thus every analytic, degenerate vacuum solution on $\mathbf{T}^{3} \times \mathbf{R}$ is diffeomorphic to one for which $\phi=\beta_{a}=0$ and $\left.\left(\frac{\partial^{n}}{\partial t^{n}} \mu_{a b}\right)\right|_{t=0}$ is covariantly constant with respect to the flat $\stackrel{\circ}{\mu}_{a b}$ for all $n>0$. Without disturbing the explicit symmetry or the vanishing of $\phi$ and $\beta_{a}$, we can perform a coordinate transformation of the form

$$
\tilde{t}=t, \quad \tilde{x}^{3}=x^{3}, \quad \tilde{x}^{a}=f^{a}\left(x^{b}\right)
$$

to bring $\stackrel{\circ}{\mu}_{a b}$ into a canonical form with constant components. In this gauge covariant constancy of the time derivatives of $\tilde{\mu}_{a b}$ at $\tilde{t}=0$ reduces to the condition that $\left.\left(\frac{\partial}{\partial \tilde{x}^{a}} \frac{\partial^{n} \tilde{\mu}_{a b}}{\partial \tilde{t}^{n}}\right)\right|_{\tilde{t}=0}=0$ which, for an analytic solution, implies that $\tilde{\mu}_{a b}=\tilde{\mu}_{a b}(\tilde{t})$. Thus we have proven:

Theorem 2. Every analytic, degenerate vacuum solution on $\mathbf{T}^{3} \times \mathbf{R}$ with simply periodic null generators is diffeomorphic to a plane wave solution. These spacetimes have compact null hypersurfaces through every event.

To complete the discussion of vacuum solutions on $\mathbf{T}^{3} \times \mathbf{R}$, we recall some results from [2] which demonstrated the existence of a large class of (nondegenerate) solutions on $\mathbf{T}^{3} \times \mathbf{R}$ by means of a slightly extended version of the Cauchy-Kowalewski theorem. The metric form considered there was given by

$$
\begin{aligned}
d s^{2}= & -\frac{e^{-2 \tilde{\phi}}}{4 t}\left(\tilde{N}^{2}-e^{4 \tilde{\phi}}\right) d t^{2}+\left(e^{-2 \tilde{\phi}} \tilde{g}_{a b}+t e^{2 \tilde{\phi}} \tilde{\beta}_{a} \tilde{\beta}_{b}\right) d x^{a} d x^{b} \\
& +\frac{e^{2 \tilde{\phi}}}{4} k^{2} t\left(d x^{3}\right)^{2}+\frac{e^{2 \tilde{\phi}}}{2} k d x^{3} d t+e^{2 \tilde{\phi}} k t \tilde{\beta}_{a} d x^{a} d x^{3}+e^{2 \tilde{\phi}} \tilde{\beta}_{a} d x^{a} d t
\end{aligned}
$$


where $k$ is a positive constant, where $\tilde{\phi}, \tilde{\beta}_{a}, \tilde{g}_{a b}$ are analytic functions of $\left\{t, x^{a}\right\}$ with $\tilde{g}_{a b}$ positive definite, and where $\tilde{N}=\left.\left(\frac{e^{2 \tilde{\phi}}}{\sqrt{{ }^{(2)} \tilde{g}}}\right)\right|_{t=0} \sqrt{{ }^{(2)} \tilde{g}}$. Again $\left\{x^{i}\right\}=\left\{x^{3}, x^{a}\right\}$ are periodic coordinates on $\mathbf{T}^{3}$ (with period $2 \pi$ ) and $\frac{\partial}{\partial x^{3}}$ is a Killing field of $g_{\mu v}$. Actually, we have slightly generalized the metric form given in [2] by letting $x^{3} \rightarrow k x^{3}$, where $k$ is a positive constant, since the previous form (with a fixed period for $x^{3}$ ) was unduly restrictive. The coordinates $\left\{t, x^{3}, x^{a}\right\}$ here correspond to the primed coordinates defined in that paper. Furthermore, the metric functions $\tilde{\phi}$, $\tilde{\beta}_{a}, \tilde{g}_{a b}$, and $\tilde{N}$ were there written without the overhead "twiddle" ( $)$. The main result of [2] was

Theorem 3. Any analytic data $\left(\tilde{\phi}, \tilde{\beta}_{a}, \tilde{g}_{a b}\right)\left(0, x^{c}\right)$, specified over $\mathbf{T}^{2}$ (with $\tilde{g}_{a b} a$ Riemannian metric) determines a unique, analytic, extendible solution of the vacuum Einstein equations on some neighborhood of the (compact, null) initial data hypersurface $t=0$. The solution covers a neighborhood of a smooth Cauchy horizon at $t=0$ and has $\frac{\partial}{\partial x^{3}}$ as a global Killing vector field. This Killing field is spacelike in the globally hyperbolic region $t>0$, null on the horizon and timelike in the acausal extension $t<0$.

We wish to prove that every non-degenerate solution is obtained by this construction. To do this we need only transform the metric form (4.28) to gaussian null coordinates with a transformation generated by

$$
\left.x^{3^{\prime}}\right|_{t=0}=x^{3},\left.\quad x^{a^{\prime}}\right|_{t=0}=x^{a},\left.\quad t^{\prime}\right|_{t=0}=0,
$$

which will preserve the explicit symmetry of the metric. A straightforward computation gives

$$
\begin{gathered}
\left.\phi^{\prime}\right|_{t^{\prime}=0}=0,\left.\quad \beta_{a}^{\prime}\right|_{t^{\prime}=0}=0,\left.\quad \phi_{, t^{\prime}}^{\prime}\right|_{t^{\prime}=0}=k, \\
\left.\beta_{a, t^{\prime}}^{\prime}\right|_{t^{\prime}=0}=\left.\left\{-2 \tilde{\phi}_{, a}+2 \tilde{\beta}_{a}\right\}\right|_{t=0}, \\
\left.\mu_{a b}^{\prime}\right|_{t^{\prime}=0}=\left.e^{-2 \tilde{\phi}_{g}} \tilde{g}_{a b}\right|_{t=0},
\end{gathered}
$$

where $\phi^{\prime}, \beta_{a}^{\prime}, \mu_{a b}^{\prime}$ represent the usual gaussian null coordinate functions in the primed coordinate system. Since $\tilde{\phi}, \tilde{\beta}_{a}$, and $\tilde{g}_{a b}$ may be specified arbitrarily at $t=0$ as may the positive constant $k$, it's clear from Eq. (4.30) that we may achieve any allowed initial values of the functions $\dot{\phi}_{, t^{\prime}}^{\prime}, \stackrel{\circ}{\beta}_{a, t^{\prime}}^{\prime}$, and $\stackrel{\circ}{\mu}_{a b}^{\prime}$. However, for a nondegenerate solution the initial value of $\mu_{a b, t^{\prime}}^{\prime}$ is determined from the Einstein equation $\stackrel{\circ}{R}_{a b}=0$ and all higher time derivatives of the metric functions $\phi^{\prime}, \beta_{a}^{\prime}$, and $\mu_{a b}^{\prime}$ are determined from successive differentiations of the field equations as shown by the proof of Theorem 1 . Therefore, we see that every non-degenerate solution (with $k>0$ ) is diffeomorphic to one constructed according to Theorem 3 . The corresponding solutions with $k<0$ may be generated by the transformation $t^{\prime} \rightarrow-t^{\prime}, x^{3^{\prime}} \rightarrow-x^{3^{\prime}}, x^{a^{\prime}} \rightarrow x^{a^{\prime}}$. Thus we have:

Theorem 4. Every non-degenerate vacuum solution on $\mathbf{T}^{3} \times \mathbf{R}$ with simply periodic null generators is diffeomorphic to one constructed according to Theorem 3. 
It follows as a corollary to Theorems 3 and 4 that the power series expressions constructed for $\phi^{\prime}, \beta_{a}^{\prime}$, and $\mu_{a b}^{\prime}$ in the proof of Theorem 1 necessarily converge. The reason is that for each allowed choice of free initial data ${\stackrel{\circ}{\phi},{ }^{\prime}}^{\prime}(=$ positive constant $)$, $\hat{\beta}_{a, t^{\prime}}^{\prime}$, and $\mu_{a b}^{\prime}$, there exists a corresponding choice [via Eq. (4.30)] of analytic data $\left(\tilde{\phi}, \tilde{\beta}_{a}, \tilde{g}_{a b}\right)$ for a solution determined according to Theorem 3 . Transforming this solution back to gaussian null coordinates by the appropriate analytic diffeomorphism, we must recover the original formal (but now necessarily convergent) series since these expressions were uniquely determined by the field equations. We thus get

Corollary 5. For any constant $\stackrel{\phi}{\phi}, t_{1}=k \neq 0$ and any analytic one-form $\stackrel{\circ}{\beta}_{a, t}$ and Riemannian metric $\stackrel{\circ}{\mu}_{a b}$ defined over $\mathbf{T}^{2}$, the formal power series expressions for $\left(\phi, \beta_{a}, \mu_{a b}\right)$ constructed in the proof of Theorem 1 are always convergent to a vacuum solution of Einstein's equation in some neighborhood of the initial surface.

It is worth remarking that the correspondence given by Eq. (4.30) is not unique since the coordinate system employed in [2] is less rigid than the gaussian null one used here.

\section{Conclusion}

The most artificial hypothesis we have made is that the generators of the compact null surfaces considered all be closed curves. We believe, however, that one should be able to eliminate this assumption and still prove the existence of a Killing symmetry in the (compact, analytic) electrovacuum case. A key first step in the needed generalization has already been provided by Hawking and Ellis [10], who show that the null generators of a compact, Cauchy horizon always have zero expansion. This corresponds to our result that $(\sqrt{\dot{\mu}})_{, 3}=0$, and leads, via Raychaudhuri's equation, to the vanishing of the shear of the generators $\left(\check{\mu}_{a b, 3}=0\right.$ in our case). The acceleration and rotation of these curves already vanish by virtue of their being the normal, geodesic generators of a null hypersurface.

These remarks suggest the intuitive picture of a congruence of "parallel" rays in the null surface $N$ which must "almost close" because of the compactness of $N$ and which locally have all the differential properties of the closed generators considered in this paper. We expect that continuity considerations should allow us to extend the arguments given here to such almost closed congruences. The main step one will have to generalize is the construction of the "surface gravity" constant $k$. The induction arguments (except for the use of the maximum principle) are essentially local and should follow the same pattern given here for the closed orbit case.

Analyticity is another artificial assumption, though a very convenient one. We would conjecture, however, that non-degenerate, non-analytic solutions with compact null surfaces of the (closed orbit) type considered here must also have symmetries and that the null surfaces must always be Killing horizons as in the analytic case. One might try to prove this by taking a completion (in say, a Sobolev topology) of the analytic, globally hyperbolic spacetimes considered here and showing that the non-analytic solutions in the completion also have non-singular 
Cauchy horizons at their future boundaries. Even if this procedure works, however, it should not be expected to produce acausal extensions of the type described here for the analytic case. The reason is that, for metrics with a Killing symmetry which changes type from spacelike to timelike across a null surface $N$, Einstein's evolution equations change type from hyperbolic to elliptic, degenerating to a parabolic system on $N$. Thus one expects from standard elliptic regularity theorems that only the analytic solutions are extendible across their horizons unless the symmetries are broken at the horizons. This symmetry breaking would presumably be possible since uniqueness of the evolution is lost in crossing a Cauchy horizon.

The main question though is not what happens beyond the horizon but rather whether the existence of a horizon implies the existence of a symmetry in the globally hyperbolic region. This, we conjecture, should still be true in the nonanalytic case.

The reader has undoubtedly noticed that the main arguments of this paper are "global" only along the null generators of $N$ and are essentially local in the transversal directions. Thus we were able to prove the existence of a symmetry on some neighborhood of an (arbitrarily small) elementary region of $N$ and only later did we patch the local symmetries together to prove the existence of a Killing field on a full neighborhood of $N$. This remark suggests the possibility of a significant extension of our results.

Suppose that $\left({ }^{(4)} V, g\right)$ is an analytic, vacuum spacetime but that ${ }^{(4)} V$ is no longer necessarily a product. Let $\left(M \times \mathbf{R},\left.g\right|_{M \times \mathbf{R}}\right)$ be a globally hyperbolic, open submanifold of $\left({ }^{(4)} V, g\right)$, and suppose that there exists an elementary region in $\left({ }^{(4)} V, g\right)$ which lies at the boundary of $\left(M \times \mathbf{R},\left.g\right|_{M \times \mathbf{R}}\right)$. The picture we have in mind is that there might not exist a compact null surface $N$ in $\left({ }^{(4)} V, g\right)$ because of the occurrence of curvature singularities along a portion of the boundary of the globally hyperbolic, open submanifold. Arguing as in this paper, we can construct a (gaussian null) neighborhood of the elementary region and prove the existence of a Killing field on this neighborhood. However, we can now appeal to analyticity and "propagate" this vector field to get a solution of Killing's equations throughout $\left({ }^{(4)} V, g\right)$.

At first glance we seem to have vastly increased the set of analytic, vacuum spacetimes which are forced to have Killing symmetries by virtue of having threedimensional submanifolds of closed null geodesics (no longer necessarily compact). There is, however, another logical possibility which we believe to be more likely - that all such analytic spacetimes necessarily have a compact null surface $N$ which contains the given elementary region. In other words, the elementary region can always be "completed" to yield a non-singular compact null surface in $\left({ }^{(4)} V, g\right)$. This conjecture (which is certainly false in the $C^{\infty}$ case) is suggested by the study of examples (certain Gowdy models [11]) which we shall not review here. If true, it represents a certain rigidity property of analytic (electro-) vacuum spacetimes.

Even though the corresponding rigidity conjecture is false for $C^{\infty}$ spacetimes [12], its validity for analytic spacetimes would apparently help one to show that non-analytic, globally hyperbolic spacetimes which have partially non-singular boundaries to their maximal Cauchy developments (containing, for example, elementary regions as mentioned above) are, in a suitable sense, always unstable. 
The reasoning would entail an argument that such "locally extendible" spacetimes could be approximated arbitrarily closely only by those analytic spacetimes which are inextendible (even locally) beyond their maximal Cauchy developments.

We believe that the conjectures mentioned above, unlike most speculation concerning the cosmic censorship conjecture, are directly amenable to analytical attack. It seems to us that a proof of these conjectures would provide strong support for the cosmic censorship idea by showing that causality violating, cosmological solutions of Einstein's equations are (at least in the electrovacuum case) essentially an artefact of symmetry.

Acknowledgement. We are grateful to A. H. Taub for helpful conversations.

\section{References}

1. Moncrief, V.: Infinite-dimensional family of vacuum cosmological models with TaubNUT(Newman-Unti-Tamburino)-type extensions. Phys. Rev. D 23, 312 (1981)

2. Moncrief, V.: Neighborhoods of Cauchy horizons in cosmological spacetimes with one Killing field. Ann. Phys. (N.Y.) 141, 83 (1982)

3. Moncrief, V.: Paper in preparation

4. See for example the articles by Geroch, R., Horowitz, G.T., Penrose, R.: In: General relativity: an Einstein centenary survey. Hawking, S., Israel. W. (eds.). Cambridge: Cambridge University Press 1979

5. Misner, C., Thorne, K., Wheeler, J. : Gravitation. San Francisco: Freeman 1973

6. See, for example: Sperb, R.: Maximum principles and their applications. New York: Academic Press 1981

To treat smooth functions on $\mathbf{S}^{1}$, one can apply the ordinary maximum principle for functions on $\mathbf{R}^{1}$ to the special case of periodic functions. For the analytic case treated in this paper one can avoid the use of the maximum principle altogether, replacing it by an inductive argument applied to the successive derivatives of Raychaudhuri's equation

7. Hawking, S., Ellis, G. : The large scale structure of space-time. Cambridge: Cambridge University Press 1973. See Proposition 6.4.4 on p. 191

8. Fischer, A., Marsden, J., Moncrief, V.: The structure of the space of solutions of Einstein's equations. I. One Killing field. Ann. Inst. H. Poincaré 33, 147 (1980). See especially Sect. (2)

9. See, for example: Carter, B. : In : Black Holes, DeWitt, C., DeWitt, B.S. (eds.). New York : Gordon and Breach 1973. See in particular Eq. (5.9) on p. 147

10. See Sect. (8.5) of Ref. [7], especially the discussion on pp. 297-298

11. The examples which suggest this "rigidity conjecture" are the polarized Gowdy models discussed in Ref. [1]

12. Moncrief, V.: Global properties of Gowdy spacetimes with $\mathbf{T}^{3} \times \mathbf{R}$ topology. Ann. Phys. (N.Y.) 132, 87 (1981). See especially Sect. IV

Communicated by S.-T. Yau

Received October 28, 1982 
